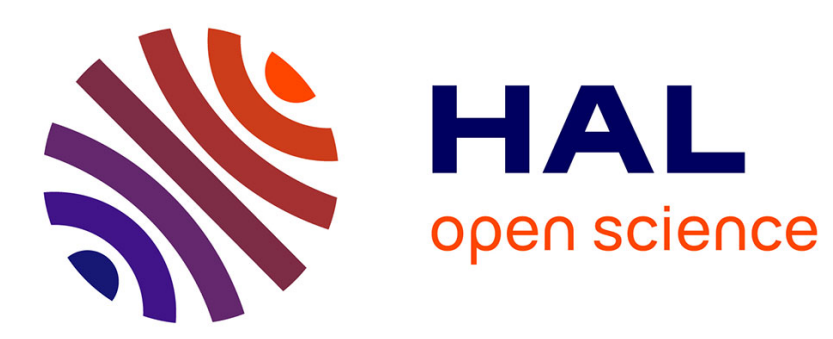

\title{
A Cramér-Rao bounds based analysis of 3D antenna array geometries made from ULA branches
}

\author{
Dinh Thang Vu, Alexandre Renaux, Remy Boyer, Sylvie Marcos
}

\section{To cite this version:}

Dinh Thang Vu, Alexandre Renaux, Remy Boyer, Sylvie Marcos. A Cramér-Rao bounds based analysis of 3D antenna array geometries made from ULA branches. Multidimensional Systems and Signal Processing, 2013, 24 (1), pp.121-155. 10.1007/s11045-011-0160-5 . hal-00769344

\section{HAL Id: hal-00769344}

https://hal-centralesupelec.archives-ouvertes.fr/hal-00769344

Submitted on 31 Dec 2012

HAL is a multi-disciplinary open access archive for the deposit and dissemination of scientific research documents, whether they are published or not. The documents may come from teaching and research institutions in France or abroad, or from public or private research centers.
L'archive ouverte pluridisciplinaire HAL, est destinée au dépôt et à la diffusion de documents scientifiques de niveau recherche, publiés ou non, émanant des établissements d'enseignement et de recherche français ou étrangers, des laboratoires publics ou privés. 


\title{
A Cramér Rao bounds based analysis of 3D antenna array geometries made from ULA branches
}

\author{
Dinh Thang VU • Alexandre RENAUX . \\ Rémy BOYER • Sylvie MARCOS
}

Received: date / Accepted: date

\begin{abstract}
In the context of passive sources localization using antenna array, the estimation accuracy of elevation, and azimuth are related not only to the kind of estimator which is used, but also to the geometry of the considered antenna array. Although there are several available results on the linear array, and also for planar arrays, other geometries existing in the literature, such as 3D arrays, have been less studied. In this paper, we study the impact of the geometry of a family of 3D models of antenna array on the estimation performance of elevation, and azimuth. The Cramér-Rao Bound (CRB), which is widely spread in signal processing to characterize the estimation performance will be used here as a useful tool to find the optimal configuration. In particular, we give closed-form expressions of CRB for a 3D antenna array under both conditional, and unconditional observation models. Thanks to these explicit expressions, the impact of the third dimension to the estimation performance is analyzed. Particularly, we give criterions to design an isotropic 3D array depending on the considered observation model. Several 3D particular geometry antennas made from uniform linear array (ULA) are analyzed, and compared with 2D antenna arrays. The isotropy condition of such arrays is analyzed. The presented framework can be used for further studies of other types of arrays.
\end{abstract}

Keywords Array geometry optimization · direction of arrival estimation · performance bound.

\section{Introduction}

In the context of passive sources localization by an array of sensors, the DirectionOf-Arrival (DOA) estimation performance is not only linked to the kind of estimator used but also to the array geometry, i.e., the sensors location in the space.

Dinh Thang VU · Alexandre RENAUX · Rémy BOYER · Sylvie MARCOS

University Paris-Sud 11, Laboratory of Signals and Systems, Supélec, 3 rue Joliot-Curie, 91192

Gif-sur-Yvette cedex, France

Tel.: +331 69851763

Fax: +331 69851765

E-mail: $\{$ Vu,Renaux,Remy.Boyer,Marcos $\} @ 1 s s . s u p e l e c . f r$ 
For an array of sensors, the meaning of "performance" can be seen from different points of view: beampattern properties, ambiguities of the array, isotropy, localization estimation in terms of mean square error (MSE), etc. A huge amount of works is available in the literature concerning the study of array ambiguities (see, e.g., $[1-5]$ ) the beampattern (see, e.g., $[6,7]$ ), and the isotropic properties of arrays (see, e.g., [8]).

In this paper, we are interested in the optimal array geometry leading to the best performance in terms of MSE. More particularly, we will focus on three dimensional (3D) array geometries less studied in the literature. Indeed, although there are already many available results on planar arrays (2D), there exists other geometries such as 3D arrays. There are many applications where the sensors are scattered in space leading to an arbitrary shape of the antenna (network of telescopes on the Earth's surface, networks of electrodes on the skull of a patient, networks of sensors in a room or in a small space for robotics functions, networks of buoys on the surface of the sea, etc). Moreover, compared to the 2D antenna, the $3 \mathrm{D}$ antenna have some intuitive advantages, such as the 3D antenna overcomes the ambiguity of the 2D antenna in some unambiguous cases. For example, one can imagine that in the radar application problem, the targets are located the 3D space and which would be hidden by certain types of landscape (hills, forests, etc.). Therefore, the targets would be "invisible" for a simple planar antenna. However, the 3D antenna could provide a better detection in this situation. The limited number of results in 3D geometry antenna is perhaps due to its complexity leading to more complex expressions.

The analysis already provided in literature deal with two kinds of geometries: geometries based on circular arrays [6] or spherical arrays [9], and geometries based on linear branches (such as the well known Uniform Linear Array (ULA), the Vshaped arrays, the cross arrays or rectangular arrays). More particular attention has been paid on uniform arrays. This paper follows the context of arrays made with ULA branches.

In order to study the performance in terms of MSE, the most popular tool is clearly the Cramér-Rao bound (CRB) [10], probably because it can generally be achieved by the variance of localization estimators for a high number of snapshots [11] or at high Signal-to-Noise Ratio (SNR) [12,13]. The CRB has already been widely used in the literature to describe the fundamental properties of arrays. Through a simple form of the CRB expression, [14] shows the impact of the sensors location on the DOA estimation accuracy in the case of $2 \mathrm{D}$ arrays. Concerning DOA estimation, in [15], [8], [16], some conditions on sensors positions to ensure the isotropy are studied for $2 \mathrm{D}$, and $3 \mathrm{D}$ arrays, by way of the off-diagonal entries of the CRB, where, the arrays have the same estimation accuracy over the whole field of view. In [17], [18], the CRB for the source position estimation based on the time difference of arrival method (TDOA) is used to prove that the best geometry which minimize the trace of the CRB matrix is the uniform angular array (UAA). A Bayesian CRB approach for the case where the source is coplanar with the antenna and the DOA is modeled as a random variable is introduced in [19]. In [20], a deep study of the CRB for 2D antenna and a source anywhere in the space has been provided, leading to interesting results concerning the so-called V-shaped array in terms of isotropy, and MSE performance. Then, based on the work of [20], a novel planar geometry called the optimum ambiguity-free planar antenna array with a closed-form of $\mathrm{V}$-shaped array has been introduced in [21]. Finally, in a 
recent work [22], the authors showed that the both conditional, and unconditional CRB, jointly with the variance of DOA obtained from MUSIC algorithm, can be expressed in the same term depending on the sensors location, and this kind of CRB expressions can be used as a tool in order to optimize the array.

Note that, in array processing, the source signal is generally modeled as a Gaussian random process or as a deterministic sequence. These models are referred to as the unconditional model, and the conditional model respectively [23]. Particularly, under conditional model, the incoming signal waveforms can be assumed as either known or unknown parameters. Consequently, the computational cost of the estimation problem varies $w . r . t$ the signal waveforms assumption. The unknown signal waveforms always leads to the increase of the parameter dimension compared to the unconditional model. On the contrary, there exist in literature several applications where the signal amplitudes are known such as mobile telecommunication. The knowledge of the signal can improve the estimation performance, also reduce the problem complexity. We can cite here several works concerning the context of known waveform signal (see, e.g., [24-28]).

Of course, since the observation model can change, there are two different CRB associated to each model called unconditional CRB (UCRB), and conditional CRB (CCRB). It has been proved that the UCRB can be achieved for a high number of snapshots [11], however, it is not achievable at high SNR (for a fixed number of snapshot) [13]. On the other hand, the CCRB is achieved at high SNR [12] but it is not achieved for a large number of snapshots [11]. Surprisingly, to the best of our knowledge, all the previously proposed results are conducted in the framework of the unconditional observation model, and consequently, in the framework of the UCRB. We will show in this paper that in the framework of the conditional model, some results concerning the array geometry differ significantly from the unconditional observation model.

In this paper, both conditional, and unconditional observation models are considered to study 3D geometries. First, we detail the Fisher Information Matrix (FIM) expressions concerning the azimuth, and elevation in the case of a general $3 \mathrm{D}$ array. The Fisher information represents the way to measure the information about the parameter contained in the observations via its likelihood function. Secondly, closed form expressions of the CRB are provided when one adds an orthogonal branch to a planar array with any geometry. This model is the first step to analyze the contribution of the third dimension where an intuitive advantage of $3 \mathrm{D}$ antenna arrays w.r.t $2 \mathrm{D}$ antenna arrays is the overcoming of the ambiguity problem in elevation estimation. Third, to analyze the impact of the array geometry on estimation, we propose several closed-form expressions of the CRB for classical array shapes made with the well known ULA branches. Note that these kinds of geometry (namely the L-shaped, and V-shaped arrays) have already been investigated in the $2 \mathrm{D}$ case, which are seen as particular cases of our proposed expressions. In [29], the L-shaped antenna arrays has been proved to have $37 \%$ better accuracy than the cross array. In [30], the author introduce the isotropic conditions for the sensors positions, and for the opening angle between the two branches of the uniform/ nonuniform V-shaped planar antenna under unconditional assumption. Our goal is to extend these geometries in the 3D case to analyze the impact of a 3D additional branch in terms of MSE. These results are then analyzed to describe the performance of these arrays in terms of MSE, isotropy, and the decoupling properties. Finally, the comparison between the 3D, 


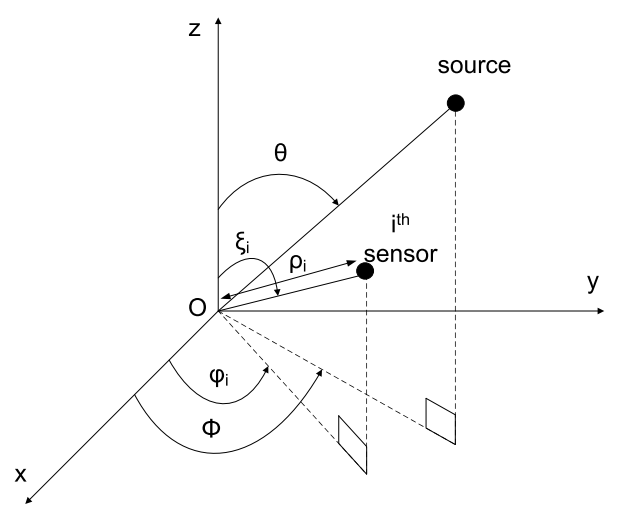

Fig. 1 Coordinate system for the source, and the sensors

and $2 \mathrm{D}$ antenna arrays, and also the comparison between $3 \mathrm{D}$, and uniform circular antenna arrays (UCA), which have the same number of sensors, are analyzed to illustrate the impact of the third dimension. However, with a constant number of sensors, in order to add the third dimension to the antenna, the aperture of the antenna must be reduced. Therefore, the estimation accuracy will be affected.

The notational convention adopted in this paper is listed as follows: italic indicates a scalar quantity, bold lower case indicates a vector, bold upper case indicates a matrix. $\widehat{\Theta}$ indicates the estimated value of $\Theta . \mathbf{A}^{T}$ is the transpose of A. $\mathbf{A}^{*}$ is the conjugate of $\mathbf{A}$. $\mathbf{A}^{H}$ is the transpose, and conjugate of $\mathbf{A}$. $\Re\{$. $\}$ denotes the real part. $\Im\{$.$\} denotes the imaginary part. |.| denotes the absolute value of$ complex scalar. $\operatorname{tr}(\mathbf{A})$ denotes the trace of matrix $\mathbf{A}$. $[\mathbf{A}]_{i, j}$ is the $\{i, j\}^{t h}$ element of matrix $\mathbf{A}$. $\operatorname{det}(\mathbf{A})$ is the determinant of the square matrix $\mathbf{A}$. $\mathbf{I}_{K}$ denotes the $K \times K$ identity matrix. And $a_{i}$ denotes the $i^{t h}$ element of vector a.

The paper is organized as follows. In Sec. II, we introduce the model, then we resume briefly the notion of CRB depending on the two hypotheses about the source signal. In Sec. III, the expressions of general CRB under the two assumptions are listed. In Sec. IV, we develop the explicit expression CRB for a family of 3D antenna arrays. Next, in Sec. V, we consider the applications of the CRB to some particular 3D antenna models to find isotropic, and uncoupling conditions. In Sec. VI, we give some typical simulations to illustrate the performance comparison between the different antenna arrays. Finally, in Sec. VII, a conclusion is presented .

\section{Model setup}

In this paper, we are interested in using an unbiased estimator to localize a single source emitting narrow-band signal in the far-field area by using a three dimensional array containing $M$ identical, and omnidirectional sensors. The source position is characterized by its spherical coordinates, such as the bearing angle vector $\Theta=[\phi \theta]^{T}$ where $\phi$ is the azimuth, and $\theta$ the elevation of the source. The $i^{t h}$ sensor position is characterized by the triple parameters $\left(\rho_{i}, \xi_{i}, \varphi_{i}\right)$ (see Fig. 1). $\theta$, and $\xi_{i}$ are measured clockwise from the $z$ axis, while $\phi$, and $\varphi_{i}$ are measured 
counter-clockwise from the $x$ axis. Letting $s(t), y(t), n(t)$ denote the source signal, the output signal at the array of sensors, and the additive noise respectively, for $t=1, \ldots, T$, where $T$ is the number of snapshots. At the $t^{t h}$ observation, the output signal at the array of sensors is then given by:

$$
\mathbf{y}(t)=\left[\begin{array}{c}
y_{1}(t) \\
\vdots \\
y_{M}(t)
\end{array}\right]=\left[\begin{array}{c}
e^{\left(\frac{j 2 \pi}{\lambda} \mathbf{v}_{1}^{T} \mathbf{r}(\Theta)\right)} \\
\vdots \\
e^{\left(\frac{j 2 \pi}{\lambda} \mathbf{v}_{M}^{T} \mathbf{r}(\Theta)\right)}
\end{array}\right] s(t)+\mathbf{n}(t)=\mathbf{a}(\boldsymbol{\Theta}) s(t)+\mathbf{n}(t)
$$

where $\lambda$ denotes the wavelength. The vector $\mathbf{a}(\boldsymbol{\Theta})$ is the $M \times 1$ steering vector with its $i^{\text {th }}$ element given by $[\mathbf{a}(\boldsymbol{\Theta})]_{i}=\exp \left(\frac{j 2 \pi}{\lambda} \mathbf{v}_{i}^{T} \mathbf{r}(\boldsymbol{\Theta})\right)$, where $\mathbf{r}(\boldsymbol{\Theta})=$ $[\sin \theta \cos \phi \sin \theta \sin \phi \cos \theta]^{T}$ is the unit vector pointing toward the source, and $\mathbf{v}_{i}=\left[\rho_{i} \sin \xi_{i} \cos \varphi_{i} \rho_{i} \sin \xi_{i} \sin \varphi_{i} \rho_{i} \cos \xi_{i}\right]^{T}$ is the position of the $i^{\text {th }}$ sensor. In the spherical coordinate system, the $i^{t h}$ element of the steering vector is given by:

$$
[\mathbf{a}(\boldsymbol{\Theta})]_{i}=e^{\left(\frac{2 j \pi \rho_{i}}{\lambda}\left(\sin \theta \sin \xi_{i} \cos \left(\phi-\varphi_{i}\right)+\cos \xi_{i} \cos \theta\right)\right)} .
$$

The noise vector $\mathbf{n}(t) \in \mathbb{C}^{M}$ is assumed to be Gaussian, circular, independent, and identically distributed (i.i.d.), zero mean with covariance matrix $\sigma_{n}^{2} \mathbf{I}_{M}$.

Concerning the source, the two following alternative hypotheses can be assumed:

- $H_{1}: s(t)$ is complex, deterministic, and assumed to be known at the receiver.

- $H_{2}: s(t)$ is assumed circular, Gaussian, zero-mean with variance $\sigma_{s}^{2}$ known at the receiver $\left(s(t) \sim \mathbb{C} \mathcal{N}\left(0, \sigma_{s}^{2}\right)\right)$, i.i.d, and independent of the noise.

Depending on the assumption $H_{1}$ or $H_{2}$ which is used, both mean or covariance matrix of the output signal may depend on $\Theta$. To be more general, let us first assume that $\mathbf{y} \mid \boldsymbol{\Theta} \sim \mathbb{C} \mathcal{N}(\boldsymbol{\mu}(\boldsymbol{\Theta}), \mathbf{R}(\boldsymbol{\Theta}))$, where $\boldsymbol{\mu}(\boldsymbol{\Theta})$ is the $M \times 1$ mean vector, and $\mathbf{R}(\boldsymbol{\Theta})$ is the $M \times M$ covariance matrix. From the Schwarz inequality, the variance of any unbiased estimator $\widehat{\boldsymbol{\Theta}}$ will satisfy: $\operatorname{var}\left(\widehat{\boldsymbol{\Theta}}_{i}\right) \geq\left[\mathbf{F I M}^{-1}(\Theta)\right]_{i i}$ which is known as the CRB, where $\operatorname{FIM}(\Theta)$ is the $M \times M$ Fisher Information Matrix (FIM). For i.i.d observations, the FIM is given by [6]:

$$
[\mathbf{F I M}(\boldsymbol{\Theta})]_{i, j}=-E\left\{\frac{\partial^{2} \ln p(\mathbf{Y} \mid \boldsymbol{\Theta})}{\partial \Theta_{i} \partial \Theta_{j}}\right\}=-\sum_{t=1}^{T} E\left\{\frac{\partial^{2} \ln p(\mathbf{y}(t) \mid \Theta)}{\partial \Theta_{i} \partial \Theta_{j}}\right\},
$$

where $\mathbf{Y}=\left[\begin{array}{lll}\mathbf{y}(1) & \ldots & \mathbf{y}(T)\end{array}\right]$. The likelihood function is given by: $p(\mathbf{Y} \mid \boldsymbol{\Theta})=$ $\prod_{t=1}^{T} p(\mathbf{y}(t) \mid \boldsymbol{\Theta})$, where

$$
p(\mathbf{y}(\mathbf{t}) \mid \boldsymbol{\Theta})=\frac{1}{\pi^{M} \operatorname{det}[\mathbf{R}(\boldsymbol{\Theta})]} \exp \left(-(\mathbf{y}(t)-\boldsymbol{\mu}(\boldsymbol{\Theta}))^{H} \mathbf{R}^{-\mathbf{1}}(\boldsymbol{\Theta})(\mathbf{y}(t)-\boldsymbol{\mu}(\boldsymbol{\Theta}))\right) .
$$

A general expression of the FIM for circular Gaussian complex observations can be deduced from [31], and [32]:

$$
[\mathbf{F I M}(\boldsymbol{\Theta})]_{i, j}=\operatorname{tr}\left(\mathbf{R}^{-1}(\Theta) \frac{\partial \mathbf{R}(\boldsymbol{\Theta})}{\partial \Theta_{i}} \mathbf{R}^{-1}(\boldsymbol{\Theta}) \frac{\partial \mathbf{R}(\boldsymbol{\Theta})}{\partial \Theta_{j}}\right)+2 \Re\left(\left[\frac{\partial \boldsymbol{\mu}(\boldsymbol{\Theta})}{\partial \Theta_{i}}\right]^{H} \mathbf{R}^{-\mathbf{1}}(\boldsymbol{\Theta}) \frac{\partial \boldsymbol{\mu}(\boldsymbol{\Theta})}{\partial \Theta_{j}}\right) .
$$


Consequently, under $H_{1}$, since $\mathbf{y}(t) \mid \boldsymbol{\Theta} \sim \mathbb{C} \mathcal{N}\left(\boldsymbol{\mu}(\boldsymbol{\Theta}), \sigma^{2} \mathbf{I}\right)$ then (4) is reduced to

$$
[\boldsymbol{F I M}(\boldsymbol{\Theta})]_{i, j}=2 \Re\left(\left[\frac{\partial \boldsymbol{\mu}(\boldsymbol{\Theta})}{\partial \Theta_{i}}\right]^{H} \mathbf{R}^{-\mathbf{1}}(\boldsymbol{\Theta}) \frac{\partial \boldsymbol{\mu}(\boldsymbol{\Theta})}{\partial \Theta_{j}}\right) .
$$

And under $H_{2}$, since $\mathbf{y}(t) \mid \boldsymbol{\Theta} \sim \mathbb{C} \mathcal{N}(\mathbf{0}, \mathbf{R}(\boldsymbol{\Theta}))$, then (4) is reduced to

$$
[\mathbf{F I M}(\boldsymbol{\Theta})]_{i, j}=\operatorname{tr}\left(\mathbf{R}^{-1}(\Theta) \frac{\partial \mathbf{R}(\boldsymbol{\Theta})}{\partial \Theta_{i}} \mathbf{R}^{-\mathbf{1}}(\boldsymbol{\Theta}) \frac{\partial \mathbf{R}(\boldsymbol{\Theta})}{\partial \Theta_{j}}\right) .
$$

The parameters of interest are the azimuth, and elevation angles, i.e., the vector $\boldsymbol{\Theta}$ which are assumed deterministic. Therefore, the $\mathrm{CRB}$, denoted $\mathbf{C}(\boldsymbol{\Theta})$, is a $2 \times 2$ matrix which can be defined as:

$$
\mathbf{C}(\boldsymbol{\Theta})=\mathbf{F I M}(\boldsymbol{\Theta})^{-1}=\left[\begin{array}{ll}
C_{\theta \theta}(\boldsymbol{\Theta}) & C_{\theta \phi}(\boldsymbol{\Theta}) \\
C_{\phi \theta}(\boldsymbol{\Theta}) & C_{\phi \phi}(\boldsymbol{\Theta})
\end{array}\right],
$$

where, $C_{\theta \theta}$, and $C_{\phi \phi}$ represent the CRBs of elevation, and azimuth, respectively. $C_{\theta \phi}=C_{\phi \theta}$ represents the coupling between parameters $\theta$, and $\phi$.

\section{FIM expressions for a general 3D array}

In this section, we will detail the CRB expressions under both the conditional, and unconditional observation models.

3.1 Conditional observation model ( $H_{1}$ assumption)

Under $H_{1}$, since the parameters only appear in the mean $\boldsymbol{\mu}(\boldsymbol{\Theta})$, i.e., $\mathbf{R}(\boldsymbol{\Theta})$ is not a function of $\Theta$ in this case, from Eqn. (4), the FIM can be simplified as follows:

$$
[\mathbf{F I M}(\boldsymbol{\Theta})]_{i, j}=2 \Re\left(\frac{\partial \boldsymbol{\mu}(\boldsymbol{\Theta})^{H}}{\partial \Theta_{i}} \mathbf{R}^{-\mathbf{1}}(\boldsymbol{\Theta}) \frac{\partial \boldsymbol{\mu}(\boldsymbol{\Theta})}{\partial \Theta_{j}}\right) .
$$

In this case, the mean vector is given by: $\boldsymbol{\mu}(\boldsymbol{\Theta})=\left(\mathbf{I}_{T} \otimes \mathbf{a}(\boldsymbol{\Theta})\right) \mathbf{s}$, where $\otimes$ denotes the Kronecker product, $\mathbf{s}$ denotes the source signal vector $s=[s(1) \ldots s(T)]^{T}$. The covariance matrix is given by: $\mathbf{R}=\sigma_{n}^{2} \mathbf{I}_{M T}$. Therefore, (6) becomes:

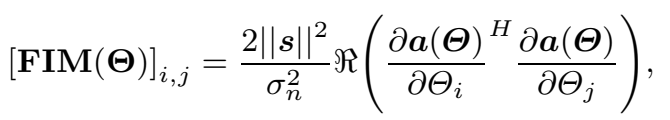

where $i, j \in\{1,2\}^{2}$, and $\Theta_{1}=\theta$, and $\Theta_{2}=\phi$, and where $\|s\|^{2}=s^{H} s$. The derivation of the steering vector w.r.t. $\theta$, and $\phi$ is

$$
\begin{array}{r}
\frac{\partial[\mathbf{a}(\boldsymbol{\Theta})]_{i}}{\partial \theta}=\frac{2 j \pi \rho_{i}}{\lambda}\left(\cos \theta \sin \xi_{i} \cos \left(\phi-\varphi_{i}\right)-\cos \xi_{i} \sin \theta\right) \\
\times e^{\left(\frac{2 j \pi \rho_{i}}{\lambda}\left(\sin \theta \sin \xi_{i} \cos \left(\phi-\varphi_{i}\right)+\cos \xi_{i} \cos \theta\right)\right)},
\end{array}
$$


and

$\frac{\partial[\mathbf{a}(\boldsymbol{\Theta})]_{i}}{\partial \phi}=\frac{2 j \pi \rho_{i}}{\lambda}\left(-\sin \theta \sin \xi_{i} \sin \left(\phi-\varphi_{i}\right)\right) e^{\left(\frac{2 j \pi \rho_{i}}{\lambda}\left(\sin \theta \sin \xi_{i} \cos \left(\phi-\varphi_{i}\right)+\cos \xi_{i} \cos \theta\right)\right)}$.

Then, (7) becomes

$$
\left\{\begin{array}{l}
\frac{[\mathbf{F I M}]_{1,1}}{C_{S N R}}=\sum_{i=1}^{M} \rho_{i}^{2}\left(\cos \theta \sin \xi_{i} \cos \left(\phi-\varphi_{i}\right)-\cos \xi_{i} \sin \theta\right)^{2}, \\
\frac{[\mathbf{F I M}]_{2,2}}{C_{S N R}}=\sum_{i=1}^{M} \rho_{i}^{2}\left(\sin \theta \sin \xi_{i} \sin \left(\phi-\varphi_{i}\right)\right)^{2}, \\
\frac{[\mathbf{F I M}]_{1,2}}{C_{S N R}}=-\sum_{i=1}^{M} \rho_{i}^{2}\left(\sin \theta \sin \xi_{i} \sin \left(\phi-\varphi_{i}\right)\right)\left(\cos \theta \sin \xi_{i} \cos \left(\phi-\varphi_{i}\right)-\cos \xi_{i} \sin \theta\right),
\end{array}\right.
$$

where $C_{S N R}=\frac{8 \pi^{2}\|s\|^{2}}{\sigma_{n}^{2} \lambda^{2}}$. And the determinant of the FIM is given by

$$
\operatorname{det}(\mathbf{F I M}(\boldsymbol{\Theta}))=[\mathbf{F I M}]_{1,1}[\mathbf{F I M}]_{2,2}-[\mathbf{F I M}]_{1,2}[\mathbf{F I M}]_{2,1} .
$$

3.2 Unconditional observation model ( $H_{2}$ assumption)

Under $H_{2}$ assumption, since the parameters only appear in the covariance $\mathbf{R}(\boldsymbol{\Theta})$, from Eqn. (4), the FIM becomes:

$$
[\mathbf{F I M}(\boldsymbol{\Theta})]_{i, j}=\operatorname{tr}\left(\mathbf{R}^{-\mathbf{1}}(\boldsymbol{\Theta}) \frac{\partial \mathbf{R}(\boldsymbol{\Theta})}{\partial \Theta_{i}} \mathbf{R}^{-\mathbf{1}}(\boldsymbol{\Theta}) \frac{\partial \mathbf{R}(\boldsymbol{\Theta})}{\partial \Theta_{j}}\right) .
$$

Because $\mathbf{R}(\boldsymbol{\Theta})=\sigma_{s}^{2} \mathbf{I}_{T} \otimes\left(\mathbf{a}(\boldsymbol{\Theta}) \mathbf{a}^{\mathbf{H}}(\boldsymbol{\Theta})\right)+\sigma_{n}^{2} \mathbf{I}_{M T}$, and from [33, eq. (39)], (12) can be written as follows:

$$
\left.[\boldsymbol{F I M}(\boldsymbol{\Theta})]_{i, j}={\frac{2 T M \sigma_{s}^{4}}{\sigma_{n}^{2}\left(\sigma_{n}^{2}+M \sigma_{s}^{2}\right)}}_{\left({\frac{\partial \boldsymbol{a}(\boldsymbol{\Theta})}{\partial \Theta_{i}}}^{H}\right.} \frac{\partial \boldsymbol{a}(\boldsymbol{\Theta})}{\partial \Theta_{j}}-\frac{1}{M}^{\frac{\partial \boldsymbol{a}(\boldsymbol{\Theta})}{\partial \Theta_{i}}}{ }^{H} \boldsymbol{a}(\boldsymbol{\Theta}) \boldsymbol{a}(\boldsymbol{\Theta})^{H}{\frac{\partial \boldsymbol{a}(\boldsymbol{\Theta})}{\partial \Theta_{j}}}^{2}\right)
$$

where $i, j=\{1,2\}^{2}$. $\frac{\partial \mathbf{a}(\boldsymbol{\Theta})}{\partial \Theta_{1}}=\frac{\partial \mathbf{a}(\boldsymbol{\Theta})}{\partial \theta}$ is given by Eqn. (8), and $\frac{\partial \mathbf{a}(\boldsymbol{\Theta})}{\partial \Theta_{2}}=\frac{\partial \mathbf{a}(\boldsymbol{\Theta})}{\partial \phi}$ is given by Eqn. (9). Then, (13) leads to (14), shown at the bottom of the page, where $U_{S N R}=\frac{8 \pi^{2} T M \sigma_{s}^{4}}{\sigma_{n}^{2} \lambda^{2}\left(\sigma_{n}^{2}+M \sigma_{s}^{2}\right)}$.

$$
\begin{aligned}
\frac{[\mathbf{F I M}]_{1,1}}{U_{S N R}}= & \sum_{i=1}^{M} \rho_{i}^{2}\left(\cos \theta \sin \xi_{i} \cos \left(\phi-\varphi_{i}\right)-\cos \xi_{i} \sin \theta\right)^{2} \\
& -\frac{1}{M}\left(\sum_{i=1}^{M} \rho_{i}\left(\cos \theta \sin \xi_{i} \cos \left(\phi-\varphi_{i}\right)-\cos \xi_{i} \sin \theta\right)\right)^{2}, \\
\frac{[\mathbf{F I M}]_{2,2}}{U_{S N R}}= & \sum_{i=1}^{M} \rho_{i}^{2}\left(\sin \theta \sin \xi_{i} \sin \left(\phi-\varphi_{i}\right)\right)^{2}-\frac{1}{M}\left(\sum_{i=1}^{M} \rho_{i} \sin \theta \sin \xi_{i} \sin \left(\phi-\varphi_{i}\right)\right)^{2}, \\
\frac{[\mathbf{F I M}]_{1,2}}{U_{S N R}}= & -\sum_{i=1}^{M} \rho_{i}^{2}\left(\cos \theta \sin \xi_{i} \cos \left(\phi-\varphi_{i}\right)-\cos \xi_{i} \sin \theta\right)\left(\sin \theta \sin \xi_{i} \sin \left(\phi-\varphi_{i}\right)\right) \\
& +\frac{1}{M} \sum_{i=1}^{M} \rho_{i}\left(\cos \theta \sin \xi_{i} \cos \left(\phi-\varphi_{i}\right)-\cos \xi_{i} \sin \theta\right) \sum_{i=1}^{M} \rho_{i} \sin \theta \sin \xi_{i} \sin \left(\phi-\varphi_{i}\right),
\end{aligned}
$$

And the determinant of the FIM is given by (11). In order to simplify the analysis of the general CRBs expressions, we will consider in the following section the CRBs expressions in case of several 3D geometries arrays based on ULA branches. 


\section{Planar array + ULA orthogonal branch}

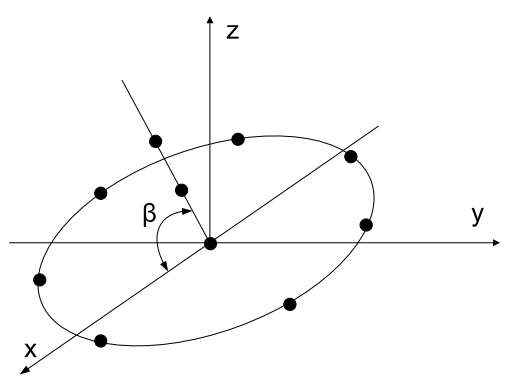

(a) Orthogonal branch antenna, and non orthogonal branch antenna

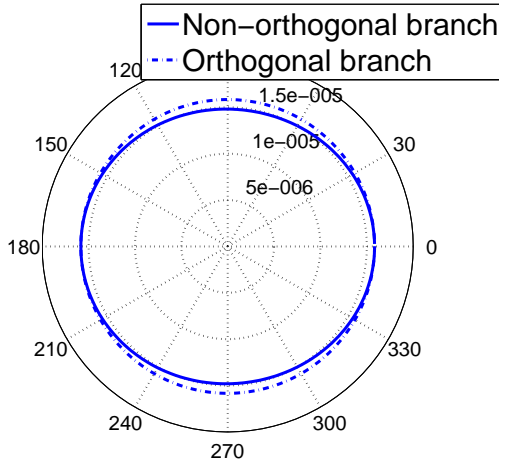

(b) $C_{\phi \phi}$ w.r.t $\phi$, with $\theta=45^{\circ}$

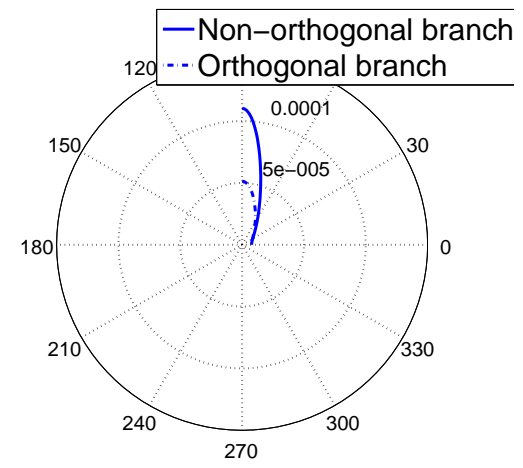

(c) $C_{\theta \theta}$ w.r.t $\theta$, with $\phi=90^{\circ}$

Fig. 2 Orthogonal branch versus non-orthogonal branch antenna.

In considering the combination of planar antenna, and the linear antenna branch in order to make an 3D antenna, there are two cases: either orthogonal branch or non-orthogonal branch. In order to analyze the impact of the array branch position to the estimation performance of the antenna, let us consider a numerical simulation about the DOA estimation performance of the antenna made from an uniform circular antenna with 7 sensors, and an uniform linear branch with 2 sensors (see Fig. 2.(a)). Let $\beta$ denotes the angle between the branch and the circular antenna plane. The inter-sensors spacing is a half-wavelength. We then compare the estimation performance between the antenna with $\beta=90^{\circ}$, i.e., orthogonal branch, and the antenna with $\beta=45^{\circ}$. Fig. 2.(b) shows the polar representation of the $\mathrm{CRB}$ of azimuth w.r.t azimuth angle with the elevation 


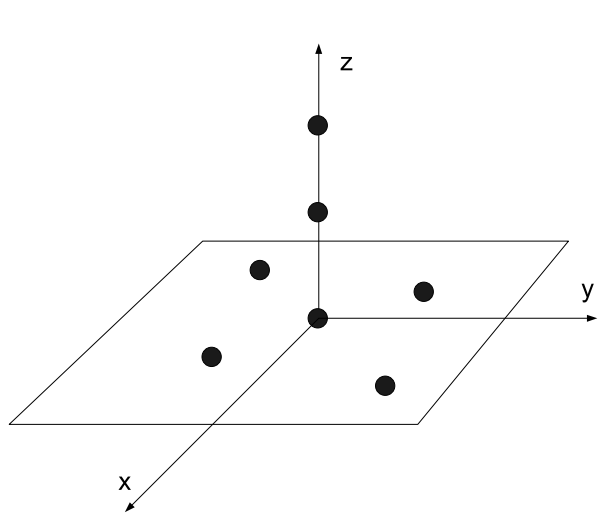

(a) Planar array with an ULA orthogonal branch

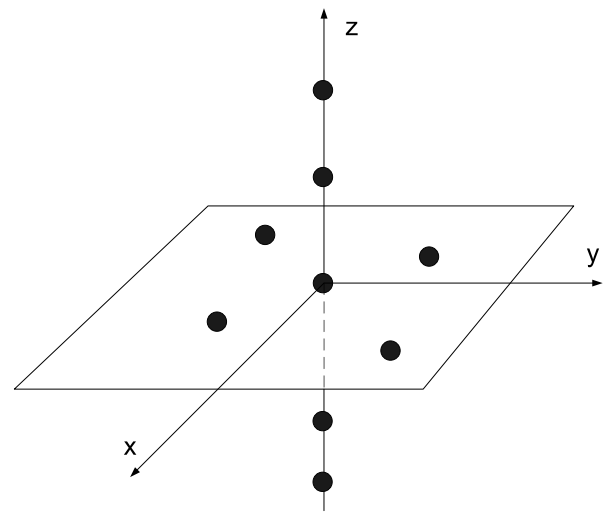

b) Planar array with two symetric ULA orthogonal branches

Fig. 3 Planar extension array

angle, $\theta=45^{\circ}$. Fig. 2.(c) represents the polar representation of the CRB of elevation w.r.t elevation angle with the azimuth angle $\phi=90^{\circ}$. The smaller the $\mathrm{CRB}$ is, the better estimation performance we have. One can observe that the antenna with orthogonal branch is better in elevation estimation accuracy than the non-orthogonal branch antenna. By several others simulations, and analyses, we observe that the compromise about the DOA estimation performance between the orthogonal and non-orthogonal branch antenna depend to the source position, i.e., the DOA. Consequently, we can not find the optimal branch position for the whole field of source position. However, let us remind that the estimation performance is strongly dependent on the aperture of the antenna. The larger the aperture antenna is, the better will be the estimation accuracy. Therefore, in order to improve the contribution of the $3^{r d}$ dimension $(\mathrm{Oz}$ direction), we have to find the sensor positioning maximizing the aperture of the antenna in the $3^{\text {rd }}$ dimension for the same aperture of the branch. It is clear that the orthogonal branch antenna is the solution. Therefore, in this paper, we consider only the orthogonal branch antenna case.

In this section, we consider an extension of an arbitrary planar array consisting of $N_{1}$ sensors when an (or two opposite) ULA orthogonal branch(es) are added. The number of sensors located on the orthogonal branch(es) is denoted by $N_{2}$. Therefore, the total number of sensors is given by $M=N_{1}+N_{2}$. Without loss of generality, let us assume that the $z$ axis is a ULA branch, while the $x O y$ plane coincides with the planar array. In order to analyze the impact of the third dimension to the estimation performance, let us denote $\rho_{k, i}$ the distance of a sensor to the origin where the index $k=1$ means that the sensor is located on the plane otherwise $k=2$ means that the sensor is located on the orthogonal branch. For this reason, $\rho_{2, i}$ represents the distance from the origin to a sensor located on the orthogonal branch, and $\rho_{1, i}$ represents the distance from the origin to a sensor 
located on the plane $x O y$. Then, let us set:

$$
\begin{aligned}
S_{12} & =\sum_{i=1}^{N_{1}} \rho_{1, i}^{2} e^{2 j \varphi_{i}}, \\
S_{10} & =\sum_{i=1}^{N_{1}} \rho_{1, i}^{2}, \\
S_{11} & =\sum_{i=1}^{N_{1}} \rho_{1, i} e^{j \varphi_{i}}, \\
S_{13} & =\sum_{i=1}^{N_{1}} \rho_{1, i}, \\
S_{20} & =\sum_{i=N_{1}+1}^{M} \rho_{2, i}^{2}, \\
S_{23} & =\sum_{i=N_{1}+1}^{M} \rho_{2, i} .
\end{aligned}
$$

Note that the parameters $S_{k, i}$, with $k \in\{1,2\}, i \in\{0, \ldots, 3\}$ depends only on the array geometry.

4.1 Planar array with an ULA orthogonal branch

The antenna geometry is illustrated in Fig 3(a).

- Conditional observation model

Under $H_{1}$, the CRB has the following compact expression (see A for the proof):

$$
\begin{aligned}
C_{\theta \theta} & =\frac{2}{C_{S N R}} \frac{\left(S_{10}-\Re\left\{S_{12} e^{-2 j \phi}\right\}\right)}{\left(\cos ^{2} \theta\left(S_{10}^{2}-\left|S_{12}\right|^{2}\right)+\sin ^{2} \theta S_{20}\left(2 S_{10}-2 \Re\left\{S_{12} e^{-2 j \phi}\right\}\right)\right)}, \\
C_{\phi \phi} & =\frac{4}{C_{S N R} \sin ^{2} \theta} \frac{\left[\frac{1}{2} \cos ^{2} \theta\left(\Re\left\{S_{12} e^{-2 j \phi}\right\}+S_{10}\right)+\sin ^{2} \theta S_{20}\right]}{\left(\cos ^{2} \theta\left(S_{10}^{2}-\left|S_{12}\right|^{2}\right)+\sin ^{2} \theta S_{20}\left(2 S_{10}-2 \Re\left\{S_{12} e^{-2 j \phi}\right\}\right)\right)}, \\
C_{\theta \phi} & =\frac{-\cos \theta}{C_{S N R} \sin \theta} \frac{\Im\left\{S_{12} e^{-2 j \phi}\right\}}{\left(\cos ^{2} \theta\left(S_{10}^{2}-\left|S_{12}\right|^{2}\right)+\sin ^{2} \theta S_{20}\left(2 S_{10}-2 \Re\left\{S_{12} e^{-2 j \phi}\right\}\right)\right)}(.15)
\end{aligned}
$$

- Unconditional observation model

The elements of the CRB is given by : $C_{i j}=\frac{N u m_{i j}}{D e n}$ where $i, j=\{\phi, \theta\}^{2}$. The denominator of CRB is given by:

$$
\begin{aligned}
\frac{D e n}{\left(U_{S N R}\right)^{2} \sin ^{2} \theta}= & \frac{\cos ^{2} \theta}{4}\left(\left(S_{10}-\frac{\left|S_{11}\right|^{2}}{M}\right)^{2}-\left|S_{12}-\frac{S_{11}^{2}}{M}\right|^{2}\right) \\
& +\frac{\sin 2 \theta}{2 M} S_{23}\left(S_{10} \Re\left\{e^{-j \phi} S_{11}\right\}-\Re\left\{e^{-j \phi} S_{12} S_{11}^{*}\right\}\right) \\
& +\sin ^{2} \theta \frac{S_{20}}{2}\left(S_{10}-\frac{\left|S_{11}\right|^{2}}{M}-\Re\left\{e^{-2 j \phi}\left(S_{12}-\frac{S_{11}^{2}}{M}\right)\right\}\right) \\
& +\sin ^{2} \theta \frac{S_{23}^{2}}{2 M}\left(\Re\left\{e^{-2 j \phi} S_{12}\right\}-S_{10}\right) .
\end{aligned}
$$

The numerators of the CRB elements are given by: 


$$
\begin{aligned}
\frac{N u m_{\phi \phi}}{U_{S N R}}= & \frac{\cos ^{2} \theta}{2}\left(S_{10}-\frac{\left|S_{11}\right|^{2}}{M}+\Re\left\{e^{-2 j \phi}\left(S_{12}-\frac{S_{11}^{2}}{M}\right)\right\}\right) \\
& +\sin ^{2} \theta\left(S_{20}-\frac{S_{23}^{2}}{M}\right)+\frac{\sin 2 \theta S_{23}}{M} \Re\left\{e^{-j \phi} S_{11}\right\}, \\
\frac{N u m_{\theta \theta}}{U_{S N R}}= & \frac{\sin ^{2} \theta}{2}\left(S_{10}-\frac{\left|S_{11}\right|^{2}}{M}-\Re\left\{e^{-2 j \phi}\left(S_{12}-\frac{S_{11}^{2}}{M}\right)\right\}\right), \\
\frac{N u m_{\theta \phi}}{U_{S N R}}= & \frac{\sin 2 \theta}{4} \Im\left\{e^{-2 j \phi}\left(S_{12}-\frac{S_{11}^{2}}{M}\right)\right\}+\frac{\sin ^{2} \theta S_{23}}{M} \Im\left\{e^{-j \phi} S_{11}\right\} .
\end{aligned}
$$

The proof are shown in B.

\subsection{Planar array with two symmetric orthogonal branches}

If the antenna structure has two symmetric orthogonal branches in such a way that the orthogonal branches centroid is located on $x O y$ plane (see Fig. 3(b)), then, a simpler CRB expression can be deduced for the unconditional model.

\section{- Conditional observation model}

In this case, the CRB has the same expressions as the previous antenna model given in Eqn. (15).

\section{- Unconditional observation model}

Let $N_{2}^{\prime}$ be the number of sensors located on the opposite orthogonal branch. Hence, the CRB expression can be deduced from the geometry described on Fig. 3(a) by letting $S_{23}=0$. This leads to:

$$
\begin{gathered}
C_{\phi \phi}=\frac{\frac{\cos ^{2} \theta\left(S_{10}-\frac{\left|S_{11}\right|^{2}}{M}+\Re\left\{e^{-2 j \phi}\left(S_{12}-\frac{S_{11}^{2}}{M}\right)\right\}\right)}{2 U_{S N R} \sin ^{2} \theta}+\frac{\sin ^{2} \theta S_{20}}{U_{S N R} \sin ^{2} \theta}}{\left(\begin{array}{l}
\frac{\cos ^{2} \theta}{4}\left(\left(S_{10}-\frac{\left|S_{11}\right|^{2}}{M}\right)^{2}-\left|S_{12}-\frac{S_{11}^{2}}{M}\right|^{2}\right) \\
+\frac{S_{20} \sin ^{2} \theta}{2}\left(S_{10}-\frac{\left|S_{11}\right|^{2}}{M}-\Re\left\{e^{-2 j \phi}\left(S_{12}-\frac{S_{11}^{2}}{M}\right)\right\}\right)
\end{array}\right)} \\
C_{\theta \theta}=\frac{\frac{1}{2 U_{S N R}}\left(S_{10}-\frac{\left|S_{11}\right|^{2}}{M}-\Re\left\{e^{-2 j \phi}\left(S_{12}-\frac{S_{11}^{2}}{M}\right)\right\}\right)}{\left(\begin{array}{l}
\frac{\cos ^{2} \theta}{4}\left(\left(S_{10}-\frac{\left|S_{11}\right|^{2}}{M}\right)^{2}-\left|S_{12}-\frac{S_{11}^{2}}{M}\right|^{2}\right) \\
+\frac{S_{20} \sin ^{2} \theta}{2}\left(S_{10}-\frac{\left|S_{11}\right|^{2}}{M}-\Re\left\{e^{-2 j \phi}\left(S_{12}-\frac{S_{11}^{2}}{M}\right)\right\}\right)
\end{array}\right)}, \\
C_{\theta \phi}=\frac{-\frac{\cos \theta}{2 U_{S N R} \sin \theta} \Im\left\{e^{-2 j \phi}\left(S_{12}-\frac{S_{11}^{2}}{M}\right)\right\}}{\left(\begin{array}{l}
\frac{\cos ^{2} \theta}{4}\left(\left(S_{10}-\frac{\left|S_{11}\right|^{2}}{M}\right)^{2}-\left|S_{12}-\frac{S_{11}^{2}}{M}\right|^{2}\right) \\
+\frac{S_{20} \sin ^{2} \theta}{2}\left(S_{10}-\frac{\left|S_{11}\right|^{2}}{M}-\Re\left\{e^{-2 j \phi}\left(S_{12}-\frac{S_{11}^{2}}{M}\right)\right\}\right)
\end{array}\right)} .
\end{gathered}
$$

See C for the proof.

\subsection{Planar array}

Due to the fact that planar array (2D) is a particular case of a $3 \mathrm{D}$ array $\left(N_{2}=0\right)$, the CRB for an arbitrary planar array are obtained by letting $S_{20}=S_{23}=0$, 
which leads to the following equations. Under $H_{1}$ assumption:

$$
\begin{aligned}
C_{\theta \theta} & =\frac{2\left(S_{10}-\Re\left\{S_{12} e^{-2 j \phi}\right\}\right)}{C_{S N R} \cos ^{2} \theta\left(S_{10}^{2}-\left|S_{12}\right|^{2}\right)}, \\
C_{\phi \phi} & =\frac{2\left(\Re\left\{S_{12} e^{-2 j \phi}\right\}+S_{10}\right)}{C_{S N R} \sin ^{2} \theta\left(S_{10}^{2}-\left|S_{12}\right|^{2}\right)}, \\
C_{\theta \phi} & =-\frac{\Im\left\{S_{12} e^{-2 j \phi}\right\}}{C_{S N R} \sin \theta \cos \theta\left(S_{10}^{2}-\left|S_{12}\right|^{2}\right)} .
\end{aligned}
$$

and under $\mathrm{H}_{2}$ assumption, the CRB leads to the results of [20]:

$$
\begin{gathered}
C_{\phi \phi}=\frac{S_{10}-\frac{\left|S_{11}\right|^{2}}{M}+\Re\left\{e^{-2 j \phi}\left(S_{12}-\frac{S_{11}^{2}}{M}\right)\right\}}{U_{S N R} \frac{\sin ^{2} \theta}{2}\left(\left(S_{10}-\frac{\left|S_{11}\right|^{2}}{M}\right)^{2}-\left|S_{12}-\frac{S_{11}^{2}}{M}\right|^{2}\right)}, \\
C_{\theta \theta}=\frac{S_{10}-\frac{\left|S_{11}\right|^{2}}{M}-\Re\left\{e^{-2 j \phi}\left(S_{12}-\frac{S_{11}^{2}}{M}\right)\right\}}{U_{S N R} \frac{\cos ^{2} \theta}{2}\left(\left(S_{10}-\frac{\left|S_{11}\right|^{2}}{M}\right)^{2}-\left|S_{12}-\frac{S_{11}^{2}}{M}\right|^{2}\right)}, \\
C_{\theta \phi}=-\frac{\left.\Im e^{-2 j \phi}\left(S_{12}-\frac{S_{11}^{2}}{M}\right)\right\}}{U_{S N R} \frac{\sin 2 \theta}{4}\left(\left(S_{10}-\frac{\left|S_{11}\right|^{2}}{M}\right)^{2}-\left|S_{12}-\frac{S_{11}^{2}}{M}\right|^{2}\right)} .
\end{gathered}
$$

\subsection{Analysis}

\subsubsection{Isotropy, and uncoupling properties}

One of several interests from the obtained closed-form expressions of the CRB is to design the array antenna in terms of isotropy, directivity, uncoupled parameters estimation... An array antenna is called isotropic if it has an uniform estimation accuracy, i.e., the CRB is not a function of the parameter of interest over the whole field of view. The uncoupled property is a desired criterion to have azimuth, and elevation estimation errors mutually independent, and hence, to avoid the degradation of the CRB. In $[1,15,16,20]$, the isotropy condition, and uncoupled parameters estimation for planar antenna was introduced. It showed that we can achieve both isotropic, and uncoupled properties with some particular array geometry. In the literature, considering isotropic property, the CRB is used only for the planar array as a criterion [8], [20], while mean square angular error (MSAE) is used for studying 3D array [8]. The CRB closed-form expressions previously derived are used here to find the array's configuration where isotropic, and/or uncoupled properties are attained.

\section{- Conditional observation model}

Because in both cases: single orthogonal branch, and two symmetric orthogonal branches, we always have the same expression for the CRB under $H_{1}$, the isotropic, and uncoupling conditions in these cases are similar. From the definition 
of isotropy, and from Eqn. (15), both isotropic (only in terms of azimuth), and uncoupling are obtained if

$$
S_{12}=0
$$

Since $S_{12}$ represents the sensors located on the plane $x O y$, we can deduce a criterion for the sensors positioning which respects to Eqn. (21):

$$
\left\{\begin{array}{l}
\sum_{i=1}^{N_{1}} \rho_{1, i}^{2} \cos 2 \varphi_{i}=0 \\
\sum_{i=1}^{N_{1}} \rho_{1, i}^{2} \sin 2 \varphi_{i}=0 .
\end{array}\right.
$$

The L-shaped array extension is an example that can achieve criterion (22), and it will be detailed in the next section.

\section{- Unconditional observation model}

For the planar antenna with a single symmetric orthogonal branch, from Eqn. (16), and (17), isotropy, and uncoupled properties can be achieved if the following expressions are both satisfied:

$$
\left\{\begin{array}{l}
S_{12}=0 \\
S_{11}=0
\end{array}\right.
$$

The expression $S_{11}=0$ leads to:

$$
\left\{\begin{array}{l}
\sum_{i=1}^{N_{1}} \rho_{1, i} \cos \varphi_{i}=0 \\
\sum_{i=1}^{N_{1}} \rho_{1, i} \sin \varphi_{i}=0
\end{array}\right.
$$

i.e., the line containing the ULA branch must pass through the centroid of the planar array. Some examples of the arrays satisfying condition (23) are shown in Fig. 4.

Contrary to the single ULA orthogonal branch case, for the planar antenna with two symmetric orthogonal branches, from Eqn. (18), isotropic, and uncoupling estimation are met if

$$
S_{12}=\frac{S_{11}^{2}}{M}
$$

It leads to the same solution of the planar arrays [20], where (23) is a particular solution. Hence, the sensors positions located on the $x O y$ plane must satisfy the following criteria:

$$
\left\{\begin{array}{l}
\sum_{i=1}^{N_{1}} \rho_{1, i}^{2} \cos 2 \varphi_{i}=\frac{\left(\sum_{i=1}^{N_{1}} \rho_{1, i} \cos \varphi_{i}\right)^{2}-\left(\sum_{i=1}^{N_{1}} \rho_{1, i} \sin \varphi_{i}\right)^{2}}{M} \\
\sum_{i=1}^{N_{1}} \rho_{1, i}^{2} \sin 2 \varphi_{i}=\frac{2}{M} \sum_{i=1}^{N_{1}} \rho_{1, i} \cos \varphi_{i} \sum_{i=1}^{N_{1}} \rho_{1, i} \sin \varphi_{i}
\end{array}\right.
$$

An intuitive solution of (25) is given by $S_{12}=S_{11}=0$ with some antenna models shown in Fig. 4 (with two symmetric orthogonal axes).

From these analysis, we can conclude here: 


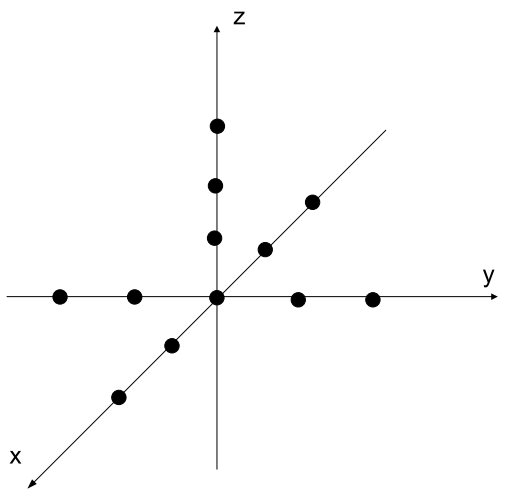

(a) Cross extension

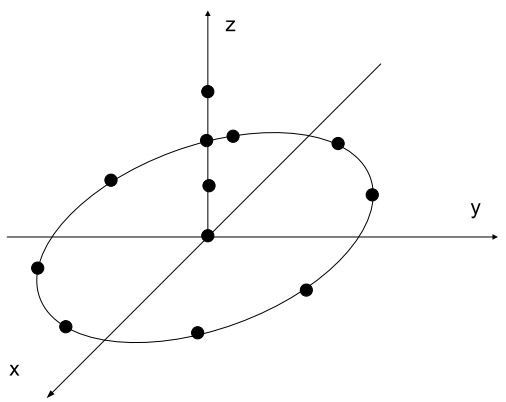

(b) Uniform circular extension

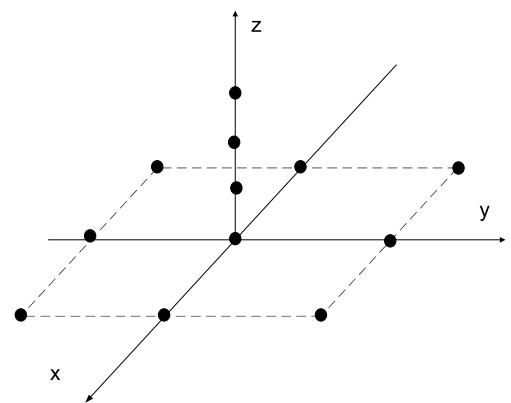

(c) Square extension

Fig. 4 Various 3D isotropic array satisfying (23)

- Under $H_{1}$, by adding an orthogonal branch to the planar antenna, or under $H_{2}$ with two symmetric branches added, the conditions of isotropy, and decoupling do not change.

- However under $H_{2}$, in the case where only one orthogonal branch is added, only the particular solution $S_{11}=S_{12}=0$ leads to the isotropy, and decoupling.

\subsubsection{Conditional versus unconditional models}

Intuitively, one can observe that the CRB expressions under $H_{1}$ are generally more compact than under $H_{2}$. Surprisingly, by comparing Eqn. (15), (16), and (17) for the 3D model, and Eqn. (19), and (20) for the planar antenna, it can be noted that: the CCRB, and the UCRB can be expressed in the same term w.r.t the sensors' location, if the following condition is satisfied:

$$
S_{11}=S_{23}=0 \text {. }
$$

In other words, the arrays will have the same behavior under both conditional, and unconditional observation models if the two ULA branches are symmetric, and the line containing these branches must pass through the centroid of the planar 
antenna. Moreover, by considering the ratio between CCRB, and UCRB for this family of arrays:

$$
\frac{U C R B}{C C R B}=\frac{C_{S N R}}{U_{S N R}}=1+\frac{1}{M \frac{\sigma_{s}^{2}}{\sigma_{n}^{2}}}=1+\frac{1}{M \times S N R},
$$

it is clear that for a large number of sensors or a high signal to noise ratio, this family of arrays has the identical estimation accuracy under both $H_{1}$, and $H_{2}$. This is consistent with the results presented in [11].

\subsection{Summary}

From these aforementioned results, some remarks can be done:

- The analytic, and compact expressions of the CRB under both conditional, and unconditional observation model for a family of 3D antenna arrays, and arbitrary $2 \mathrm{D}$ antenna arrays are derived.

- The CRB of azimuth, and elevation of the 2D models are a cosine or a sine function of the source elevation. This has been already noticed in [20] for the unconditional case, but, to the best of our knowledge, was not known in the conditional observation case. They vary in opposite ways: when the azimuth CRB is minimum, the elevation CRB is maximum, and conversely. Moreover, one can see that the CRBs of azimuth (respectively elevation) tends to infinity when elevation tends to $0^{\circ}$ (respectively $90^{\circ}$ ). However, the CRB of elevation of the $3 \mathrm{D}$ arrays is no longer a sine function of elevation, and has a finite value at $\theta=90^{\circ}$. Consequently, the $3 \mathrm{D}$ arrays model overcomes the ambiguity problem case of the $2 \mathrm{D}$ arrays.

- We found the conditions on the array geometry, with which we obtain the same estimation accuracy under both $H_{1}$, and $H_{2}$ assumptions.

- The isotropic, and decoupling criterions are introduced. We found that, under $H_{1}$, adding an orthogonal branch to the planar array does not change the conditions of isotropy, and decoupling. While under $\mathrm{H}_{2}$, depending to the number of branches added (single branch or two symmetric branches), the conditions of isotropy, and decoupling may be modified then leading to a particular solution.

\section{Particular cases}

In the previous Section, an array geometry consisting of a single orthogonal branch (or two symmetric orthogonal branches) added to an arbitrary planar array has been considered, and closed-forms expressions of CRB have been introduced. In this Section, we will detail these CRB expressions for several important particular cases of planar antennas, and their 3D extensions in order to simplify the antenna design problem. These antenna array geometries have been widely studied in several works but almost all of them are limited to the $2 \mathrm{D}$ geometry arrays. In particular, the $3 \mathrm{D}$ extension of the $\mathrm{V}$-shaped antenna array will be used here to analyze the impact of the third dimension on the estimation accuracy. 


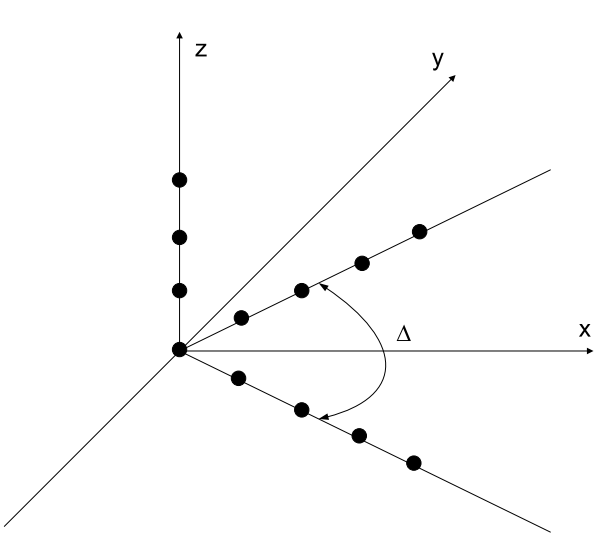

(a) V-shaped with an orthogonal branch

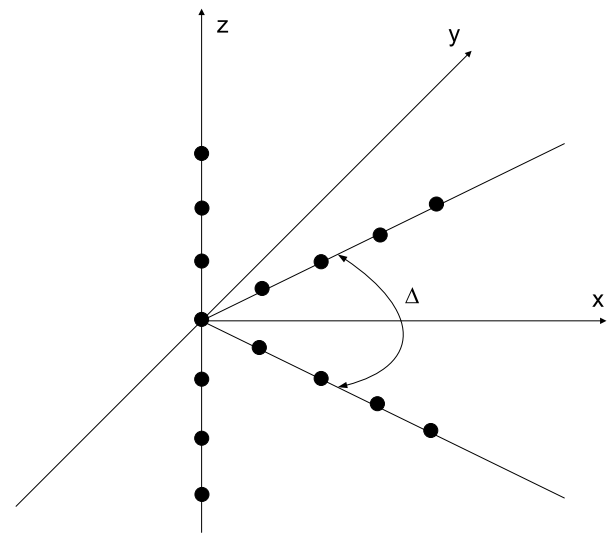

(b) V-shaped with two orthogonal symmetric branch

Fig. 5 V-shaped extension array

\subsection{D extension of the V-shaped array}

First of all, we study the $\mathrm{V}$-shaped array extension consisting of a $2 \mathrm{D} \mathrm{V}$-shaped array made from two ULA branches separated by an angle denoted $\Delta$, and from one or two opposite ULA orthogonal branches (Fig. 5). Without loss of generality, we assume that the $\mathrm{V}$-shaped array is located on the $x O y$ plane, while its ULA orthogonal branch(es) coincide(s) with the $z$ axis. The opening angle $\Delta$ is used as a degree of freedom to find the optimal geometry. Note that in [20], the Vshaped 2D array has been studied only under the unconditional observation model. Consequently, a condition on $\Delta$ leading to an isotropic array when the number of sensors $M$ tends to infinity was found: $\left(\Delta_{i s o}=2 \arctan (1 / 2)\right)$. The authors proved also that the $\mathrm{V}$-shaped $2 \mathrm{D}$ array has better performance than the classical uniform circular array for the same number of sensors.

Consequently, we here extend the work of [20] to the 3D case under both conditional, and unconditional models. For this array, under both assumptions $H_{1}$, and $H_{2}$, the parameters $S_{12}, S_{11}, S_{10}$ can be expressed as:

$$
\left\{\begin{array}{l}
S_{12}=S_{10} \cos \Delta, \\
S_{11}=S_{13} \cos \frac{\Delta}{2}, \\
\Im\left\{S_{12} e^{-2 j \phi}\right\}=-S_{10} \cos \Delta \sin 2 \phi, \\
\Re\left\{S_{12} e^{-2 j \phi}\right\}=S_{10} \cos \Delta \cos 2 \phi, \\
\Re\left\{S_{11} e^{-j \phi}\right\}=S_{13} \cos \frac{\Delta}{2} \cos \phi, \\
\Im\left\{S_{11} e^{-j \phi}\right\}=-S_{13} \cos \frac{\Delta}{2} \sin \phi, \\
\Re\left\{e^{-2 j \phi}\left(S_{12}-\frac{S_{11}^{2}}{M}\right)\right\}=\left(S_{10} \cos \Delta-\frac{S_{13}^{2} \cos ^{2} \frac{\Delta}{2}}{M}\right) \cos 2 \phi, \\
\Re\left\{e^{-j \phi} S_{12} S_{11}^{*}\right\}=S_{10} S_{13} \cos \Delta \cos \frac{\Delta}{2} \cos \phi .
\end{array}\right.
$$

These parameters will be then applied into Eqn. (15), (16), (17), and (18) in order to find closed-form expressions of the CRB of the $\mathrm{V}$-shaped 3D array extension. 
5.1.1 V-shaped 2D array with an orthogonal branch

The geometry of this antenna model is presented in Fig. 5(a).

- Conditional observation model

The CRB is easily derived from Eqn. (15), and leads to

$$
\begin{aligned}
C_{\theta \theta} & =\frac{2}{C_{S N R}} \frac{S_{10}(1-\cos \Delta \cos 2 \phi)}{\left(S_{10}^{2} \sin ^{2} \Delta \cos ^{2} \theta+\sin ^{2} \theta 2 S_{10} S_{20}(1-\cos \Delta \cos 2 \Phi)\right)} \\
C_{\phi \phi} & =\frac{4}{C_{S N R} \sin ^{2} \theta} \frac{\left(\frac{1}{2} \cos ^{2} \theta S_{10}(\cos \Delta \cos 2 \phi+1)+\sin ^{2} \theta S_{20}\right)}{\left(S_{10}^{2} \sin ^{2} \Delta \cos ^{2} \theta+\sin ^{2} \theta 2 S_{10} S_{20}(1-\cos \Delta \cos 2 \Phi)\right)} \\
C_{\theta \phi} & =\frac{1}{C_{S N R} \tan \theta} \frac{S_{10} \cos \Delta \sin 2 \phi}{\left(S_{10}^{2} \sin ^{2} \Delta \cos ^{2} \theta+\sin ^{2} \theta 2 S_{10} S_{20}(1-\cos \Delta \cos 2 \Phi)\right)}
\end{aligned}
$$

- Unconditional observation model

By applying Eqn. (29) into Eqn. (16), and (17), the CRB is given by: $C_{i j}=\frac{N u m_{i j}}{D e n}$ where $(i, j)=\{\theta, \phi\}$, and where the denominator Den is given by

$$
\begin{aligned}
\frac{\text { Den }}{\left(U_{S N R}\right)^{2} \sin ^{2} \theta}= & \cos ^{2} \theta S_{10}\left(\frac{S_{10} \sin ^{2} \Delta}{4}+\frac{S_{13}^{2} \cos ^{2} \frac{\Delta}{2}}{2 M}(\cos \Delta-1)\right) \\
& +\frac{\sin 2 \theta S_{23} S_{10} S_{13}}{2 M} \cos \frac{\Delta}{2}(1-\cos \Delta) \cos \phi \\
& +\sin ^{2} \theta \cos 2 \phi\left(\frac{S_{23}^{2} S_{10} \cos \Delta}{2 M}-\frac{S_{20}}{2}\left(S_{10} \cos \Delta-\frac{S_{13}^{2} \cos ^{2} \frac{\Delta}{2}}{M}\right)\right) \\
& +\sin ^{2} \theta\left(\frac{S_{20} S_{10}}{2}-\frac{S_{20} S_{13}^{2} \cos ^{2} \frac{\Delta}{2}}{2 M}-\frac{S_{10} S_{23}^{2}}{2 M}\right) .
\end{aligned}
$$

and where the numerators are given by

$$
\begin{aligned}
\frac{N u m_{\phi \phi}}{U_{S N R}}= & \cos ^{2} \theta\left(\frac{S_{10}}{2}-\frac{S_{13}^{2} \cos ^{2} \frac{\Delta}{2}}{2 M}\right)+\sin ^{2} \theta\left(S_{20}-\frac{S_{23}^{2}}{M}\right) \\
& +\frac{\cos ^{2} \theta \cos 2 \phi}{2}\left(S_{10} \cos \Delta-\frac{S_{13}^{2} \cos ^{2} \frac{\Delta}{2}}{M}\right) \\
& +\frac{1}{M} \sin 2 \theta S_{23} S_{13} \cos \frac{\Delta}{2} \cos \phi, \\
\frac{N u m_{\theta \theta}}{U_{S N R}}= & \sin ^{2} \theta\left(\frac{S_{10}}{2}-\frac{S_{13}^{2} \cos ^{2} \frac{\Delta}{2}}{2 M}\right)-\frac{\sin ^{2} \theta \cos 2 \phi}{2}\left(S_{10} \cos \Delta-\frac{S_{13}^{2} \cos ^{2} \frac{\Delta}{2}}{M}\right), \\
\frac{N u m_{\theta \phi}}{U_{S N R}}= & -\frac{\sin 2 \theta}{4} \sin 2 \phi\left(S_{10} \cos \Delta-\frac{S_{13}^{2} \cos ^{2} \frac{\Delta}{2}}{M}\right)-\frac{\sin ^{2} \theta}{M} S_{23} S_{13} \cos \frac{\Delta}{2} \sin \phi .
\end{aligned}
$$

The analysis of these expressions will be detailed in the next section. 
5.1.2 $V$-shaped $2 D$ array with two symmetric orthogonal branches

The geometry of this antenna model is presented in Fig. 5(b).

- Conditional observation model

The expressions of CRB under $H_{1}$ are the same as Eqn. (30).

\section{- Unconditional observation model}

Similarly to the above section, if the 3D array is built from a planar array, and two orthogonal symmetric branches (Fig. 5(b)), by applying Eqn. (29) into Eqn. (18), we have a more compact CRB expressions given by:

$$
\begin{aligned}
& C_{\phi \phi}=\frac{\frac{\cos ^{2} \theta\left(\frac{S_{10}}{2}-\frac{S_{13}^{2} \cos ^{2} \frac{\Delta}{2}}{2 M}\right)+\sin ^{2} \theta S_{20}+\frac{1}{2} \cos ^{2} \theta \cos 2 \phi\left(S_{10} \cos \Delta-\frac{S_{13}^{2} \cos ^{2} \frac{\Delta}{2}}{M}\right)}{U_{S N R} \sin ^{2} \theta}}{\left(\begin{array}{l}
S_{10} \cos ^{2} \theta\left(\frac{S_{10} \sin ^{2} \Delta}{4}+\frac{S_{13}^{2} \cos ^{2} \frac{\Delta}{2}(\cos \Delta-1)}{2 M}\right) \\
-S_{20} \sin ^{2} \theta\left(\cos 2 \phi\left(S_{10} \cos \Delta-\frac{S_{13}^{2} \cos ^{2} \frac{\Delta}{2}}{M}\right)-\left(\frac{S_{10}}{2}-\frac{S_{13}^{2} \cos ^{2} \frac{\Delta}{2}}{2 M}\right)\right)
\end{array}\right)} \\
& C_{\theta \theta}=\frac{\frac{S_{10}-\frac{1}{M} S_{13}^{2} \cos ^{2} \frac{\Delta}{2}-\cos 2 \phi\left(S_{10} \cos \Delta-\frac{S_{13}^{2} \cos ^{2} \frac{\Delta}{2}}{M}\right)}{2 U_{S N R}}}{\left(\begin{array}{l}
S_{10} \cos ^{2} \theta\left(\frac{S_{10} \sin ^{2} \Delta}{4}+\frac{S_{13}^{2} \cos ^{2} \frac{\Delta}{2}(\cos \Delta-1)}{2 M}\right) \\
-S_{20} \sin ^{2} \theta\left(\cos 2 \phi\left(S_{10} \cos \Delta-\frac{S_{13}^{2} \cos ^{2} \frac{\Delta}{2}}{M}\right)-\left(\frac{S_{10}}{2}-\frac{S_{13}^{2} \cos ^{2} \frac{\Delta}{2}}{2 M}\right)\right)
\end{array}\right)}, \\
& C_{\theta \phi}=\frac{\frac{\sin ^{2} \theta \cos \theta \sin 2 \phi\left(S_{10} \cos \Delta-\frac{S_{13}^{2} \cos ^{2} \frac{\Delta}{2}}{M}\right)}{2 U_{S N R}}}{\left(\begin{array}{l}
S_{10} \cos ^{2} \theta\left(\frac{S_{10} \sin ^{2} \Delta}{4}+\frac{S_{13}^{2} \cos ^{2} \frac{\Delta}{2}(\cos \Delta-1)}{2 M}\right) \\
-S_{20} \sin ^{2} \theta\left(\cos 2 \phi\left(S_{10} \cos \Delta-\frac{S_{13}^{2} \cos ^{2} \frac{\Delta}{2}}{M}\right)-\left(\frac{S_{10}}{2}-\frac{S_{13}^{2} \cos ^{2} \frac{\Delta}{2}}{2 M}\right)\right)
\end{array}\right)} .
\end{aligned}
$$

These expressions concerning the V-shaped 3D array under conditional, and unconditional observation models will be analyzed in the next section.

\subsection{L-shaped 3D array extension}

We call "L-shaped 3D array extension" a particular case of the V-shaped 3D array where the parameter $\Delta$ is fixed to be $\Delta=\frac{\pi}{2}$. The L-shaped (2D) array has already been studied in [29] where it is shown that the L-shaped (2D) array is $37 \%$ better in terms of estimation accuracy than the cross array. Without loss of generality, let us suppose that the three branches of the array coincides with the coordinate system axes (see Fig. 6(a)). 


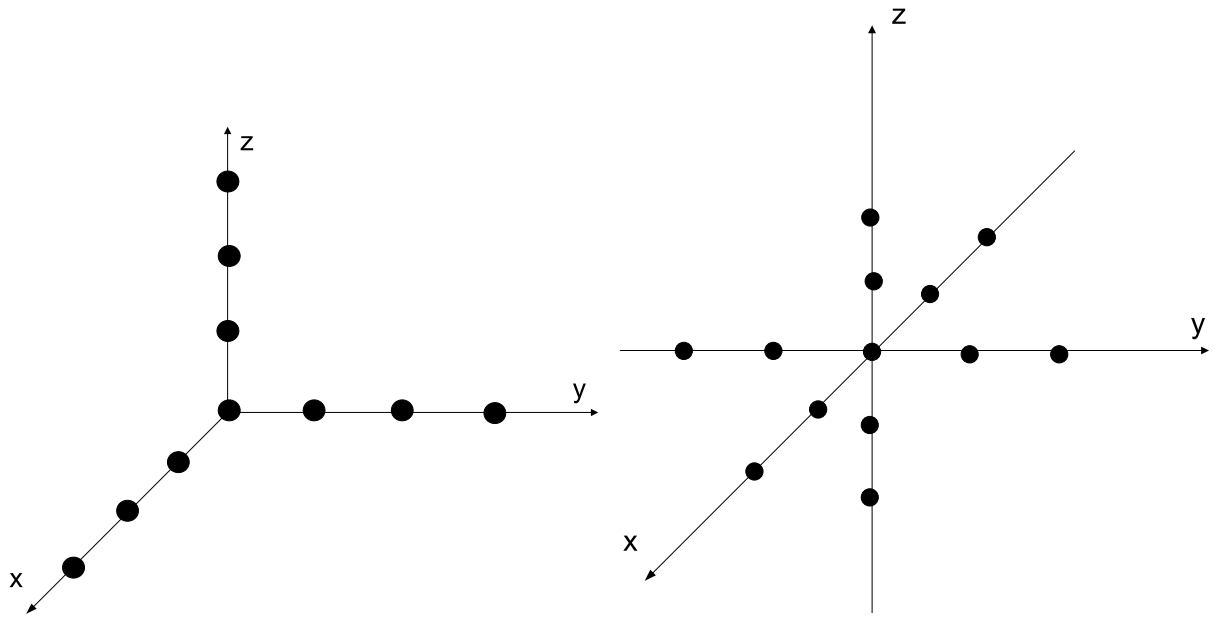

(a) L-shaped 3D array extension

(b) 3D uniform angular array

Fig. 6 Orthogonal arrays

\subsubsection{Conditional observation model}

Under $H_{1}$, expression (30) leads to

$$
\begin{aligned}
C_{\theta \theta} & =\frac{2}{C_{S N R}\left(S_{10} \cos ^{2} \theta+2 S_{20} \sin ^{2} \theta\right)}, \\
C_{\phi \phi} & =\frac{2}{C_{S N R} S_{10} \sin ^{2} \theta}, \\
C_{\theta \phi} & =0 .
\end{aligned}
$$

We can notice that, in this case, the parameters $\theta$, and $\phi$ are decoupled. The CRB becomes very compact. 


\subsubsection{Unconditional observation model}

Under $H_{2}$, by letting $\Delta=90^{\circ}$, Eqn. (31), and (32) become

$$
\begin{aligned}
& C_{\phi \phi}=\frac{\frac{\left(\cos ^{2} \theta\left(\frac{S_{10}}{2}-\frac{S_{13}^{2}(\cos 2 \phi+1)}{4 M}\right)+\sin ^{2} \theta\left(S_{20}-\frac{S_{23}^{2}}{M}\right)+\frac{1}{M \sqrt{2}} \sin 2 \theta \cos \phi S_{23} S_{13}\right)}{U_{S N R} \sin ^{2} \theta}}{\left(\begin{array}{l}
S_{10} \cos ^{2} \theta\left(\frac{S_{10}}{4}-\frac{S_{13}^{2}}{4 M}\right)+\frac{S_{23} S_{10} S_{13} \cos \phi \sin 2 \theta}{2 \sqrt{2} M} \\
-\frac{1}{4 M} \sin ^{2} \theta \cos 2 \phi S_{20} S_{13}^{2}+\sin ^{2} \theta\left(\frac{S_{20} S_{10}}{2}-\frac{S_{20} S_{13}^{2}}{4 M}-\frac{S_{10} S_{23}^{2}}{2 M}\right)
\end{array}\right)} \\
& C_{\theta \theta}= \frac{\frac{1}{2 U_{S N R}}\left(S_{10}+\frac{1}{2 M} S_{13}^{2}(\cos 2 \phi-1)\right)}{\left(\begin{array}{l}
S_{10} \cos ^{2} \theta\left(\frac{S_{10}}{4}-\frac{S_{13}^{2}}{4 M}\right)+\frac{S_{23} S_{10} S_{13} \cos \phi \sin 2 \theta}{2 \sqrt{2} M} \\
-\frac{1}{4 M} \sin ^{2} \theta \cos 2 \phi S_{20} S_{13}^{2}+\sin ^{2} \theta\left(\frac{S_{20} S_{10}}{2}-\frac{S_{20} S_{13}^{2}}{4 M}-\frac{S_{10} S_{23}^{2}}{2 M}\right)
\end{array}\right)}, \\
& C_{\theta \phi}=\frac{\left.\begin{array}{l}
\frac{1}{\sqrt{2} M U_{S N R} \sin ^{2} \theta}\left(\frac{1}{4 \sqrt{2}} S_{13}^{2} \sin _{2} \theta \sin 2 \phi-\sin ^{2} \theta \sin \phi S_{23} S_{13}\right) \\
S_{10} \cos ^{2} \theta\left(\frac{S_{10}}{4}-\frac{S_{13}^{2}}{4 M}\right)+\frac{S_{23} S_{10} S_{13} \cos \phi \sin 2 \theta}{2 \sqrt{2} M} \\
-\frac{1}{4 M} \sin ^{2} \theta \cos 2 \phi S_{20} S_{13}^{2}+\sin ^{2} \theta\left(\frac{S_{20} S_{10}}{2}-\frac{S_{20} S_{13}^{2}}{4 M}-\frac{S_{10} S_{23}^{2}}{2 M}\right)
\end{array}\right)}{} .
\end{aligned}
$$

\subsection{D uniform angular array}

A natural variant of "L-shaped 3D extension array", presented in Fig. 6(b), can be considered. This array is called 3D uniform angular antenna array (UAA). In [17], the UAA has been proved that it minimizes the CRB for the case of source position's estimation. Thanks to its special structure, which is totally symmetric, its CRB becomes more compact due to the fact that $S_{11}=S_{12}=S_{13}=S_{23}=0$, so we obtain:

\subsubsection{Conditional observation model}

The CRB is the same as Eqn. (34).

\subsubsection{Unconditional observation model}

$$
\left\{\begin{array}{l}
C_{\phi \phi}=\frac{2}{U_{S N R} S_{10} \sin ^{2} \theta}, \\
C_{\theta \theta}=\frac{2}{U_{S N R}\left(S_{10} \cos ^{2} \theta+2 S_{20} \sin ^{2} \theta\right)}, \\
C_{\theta \phi}=0 .
\end{array}\right.
$$

From Eqn. (34), and (36), we observe that under $H_{1}$, and $H_{2}$, the CRB of the UAA has identical expressions except the terms $C_{S N R}$ under $H_{1}$, and $U_{S N R}$ under $H_{2}$. Therefore, we conclude that the UAA has the similar behaviors under both conditional, and unconditional observation assumptions.

Moreover, if we choose the array structure such as $S_{20}=\frac{S_{10}}{2}$, i.e., the number of sensors of the six branches are equal, or $N_{2}=N_{2}^{\prime}=\frac{N_{1}-1}{4}$, then the CRB of elevation is independent to both the azimuth, and elevation, i.e., to DOA. 


\subsection{Analysis}

In this Section, the aforementioned results for the particular antenna models are analyzed in order to find the isotropy, uncoupling condition, and also to compare their behavior under the conditional, and unconditional assumptions.

\subsubsection{Isotropy, and uncoupling properties}

In this case, our purpose is to find the value of the degree of freedom $\Delta_{i s o}$ with which, the V-shaped extension arrays attain isotropy, and/ or decoupling.

\section{- Conditional observation model}

The condition of isotropy, and decoupling (21) leads Eqn. (30) to $\Delta_{\text {iso }}=90^{\circ}$ for both V-shaped with a single orthogonal branch or with two symmetric orthogonal branches antenna. It can be noted that this case is in contradiction with the results mentioned in [20] for the unconditional model, and 2D array, and with the results obtained below.

\section{- Unconditional observation model}

Concerning the V-shaped array with an orthogonal branch, from condition (23), the isotropic property is achieved if $S_{11}=0$ is satisfied, i.e., the line containing the ULA branch must pass through the centroid of the planar antenna. Given the fact that the line containing the ULA branch does not pass through the centroid of the planar part of the V-shaped 3D extension, therefore, there does not exist any value of $\Delta$ satisfying the isotropic condition.

Concerning the V-shaped array with two symmetric orthogonal branches, from Eqn. (33), and (25), we can see that $\Delta_{i s o}$ is the solution of equation $S_{12}-\frac{S_{11}^{2}}{M}=0$. Consequently, depending on the method used to make the branches of the antenna array (ULA, minimum redundancy [34], D-optimal [35], etc.) we might obtain different values of $\Delta_{i s o}$. In the case where the antenna array is made from ULA, then from (26), it easily leads to:

$$
\Delta_{i s o}=\arccos \left(\frac{3\left(N_{1}^{2}-1\right)}{8 M N_{1}-3 N_{1}^{2}+3}\right) .
$$

Let us set the positive $\alpha=\frac{N_{1}}{M} \leq 1$. The value of $\alpha$ associated to a planar antenna will be equal to 1 , while that one associated to a $3 \mathrm{D}$ antenna array is strictly lower than 1 . Then, $\Delta_{i s o}$ can be expressed as $\Delta_{i s o}=\arccos \left(\frac{3\left(\alpha^{2}-1 / M^{2}\right)}{8 \alpha-3 \alpha^{2}+3 / M^{2}}\right)$. We are interested also to define the range of $\Delta_{i s o}$ w.r.t. $\alpha$ in this case. It is clear that:

$$
\begin{aligned}
& \text { If } \alpha \rightarrow 1, \text { and } M \gg 1 \Rightarrow \Delta_{i s o} \simeq \arccos \left(\frac{3}{5}\right)=53.13^{\circ} \\
& \text { If } \alpha \rightarrow 0, \text { and } M \gg 1 \Rightarrow \Delta_{\text {iso }} \simeq \arccos (0)=90^{\circ}
\end{aligned}
$$

In Fig. 7, when $\alpha$ tends to 0, i.e, the number of sensors located on the orthogonal axis is much larger than the number of sensors located on the planar array, then, the value of $\Delta_{i s o}$ tends to $90^{\circ}$. On the contrary, if $\alpha$ tends to 1 , i.e, the number of sensors located on the planar array is much larger than those located on the orthogonal axis, then, the value of $\Delta_{i s o}$ tends to $\arccos (3 / 5)$. In particular, in the case where $\alpha=1$, we obtain exactly the same result $\left(\Delta_{i s o}=53.13^{\circ}\right)$ for the 


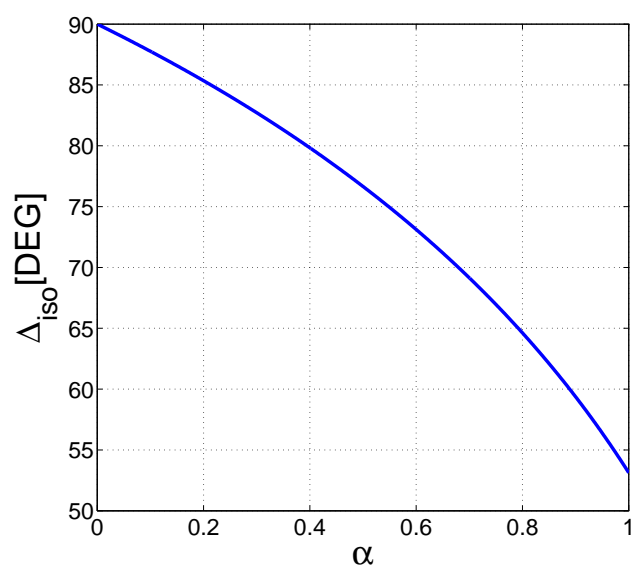

Fig. 7 Variation of $\Delta_{\text {iso }}$ w.r.t. $\alpha$ with $M=1000$.

planar antenna array as in [20]. Therefore, $\Delta_{i s o}$ in this case will vary from $53.13^{\circ}$ to $90^{\circ}$.

A remark can be done here that under $H_{2}$, adding two symmetric orthogonal branches does not modify the conditions of isotropic, and decoupling $\left(S_{12}=\right.$ $\left.S_{11}^{2} / M\right)$ w.r.t the planar array, but it changes the arrangement of the sensors located on the planar part because of the intervention of $N_{2}$ to $S_{12}$, and $S_{11}$.

\subsubsection{Conditional versus unconditional models}

Since the V-shaped 3D extension array does not satisfy condition (27) because the the line containing the ULA branch does not pass through the centroid of the planar part of the antenna, then it is impossible to find an optimal value of $\Delta$, with which, the CCRB, and the UCRB have the same expressions. The CCRB in this case is always more compact than the UCRB. Contrary to the V-shaped 3D extension array, the 3D UAA satisfies well condition (27), therefore the UCRB, and CCRB will have the identical compact expression at high $S N R$ or for a large number of sensors.

\subsection{Summary}

Thanks to the degree of freedom $\Delta$ of the V-shaped family arrays, the analysis of the impact of the array geometry on the estimation performance is simplified. We here can make some remarks:

- In almost cases, CCRB has a more compact expression than UCRB.

- Under $H_{1}$, the value of $\Delta_{i s o}$ is constant $\left(\Delta_{i s o}=90^{\circ}\right)$, while it takes a range of values under $H_{2}$, depending to the antenna array configuration. In particular, when $\alpha=1$, we find the same results $\left(\Delta_{i s o}=53.13^{\circ}\right)$ for the $\mathrm{V}$-shaped $(2 \mathrm{D})$ antenna as in [20]. 
- The 3D uniform angular array has several advantages: isotropy, uncoupling, minimization of the CRB in case of the source position's location using TDOA method, and the same estimation accuracy under both the $H_{1}$ or $H_{2}$ assumptions.

\section{Comparison of the estimation accuracy}

In this section, we will use the closed form expressions of the CRB calculated in the previous section to compare the estimation performance between the above studied arrays with other classical arrays. In order to simplify the array design problem, we only consider the behavior of the CRB of the $\mathrm{V}$-shaped antenna array and its $3 \mathrm{D}$ extension. Its closed form CRB will be analyzed w.r.t. the opening angle $\Delta$. For the simulation, all branches of the antenna array being 2D (two branches) either 3D (three branches or four branches) are made from ULAs with the inter-sensor space of half the wavelength. The simulations are performed with a signal to noise ratio equal to $10 d B$ and a number of snapshots $T=100$.

6.1 Comparison of the estimation performance between the V-shaped 3D extension antenna array and the planar circular antenna array

We here compare the estimation performance between the $\mathrm{V}$-shaped antenna array with an isotropic classic antenna: the uniform circular antenna (UCA). For this comparison, the antenna arrays will have the same number of sensors. The sensors of UCA are half-wavelength inter-element spaced, thus, the value of its radius is given by $r=\frac{\lambda}{4 \sin \frac{\pi}{M}}$. Figs. 8 and 9 represent respectively the $\mathrm{CRB}$ of azimuth and elevation normalized by the $\mathrm{CRB}$ of the $\mathrm{UCA}\left(C_{\theta \theta} / C_{\theta \theta}^{(U C A)}, C_{\phi \phi} / C_{\phi \phi}^{(U C A)}\right)$ w.r.t. the aforementioned coefficient $\alpha$, at the opening angle $\Delta=60^{\circ}$ and at the elevation $\theta=45^{\circ}$ under both conditional and unconditional observation models.

- Conditional observation model

In Fig 9(a), the accuracy concerning the elevation estimation of the $\mathrm{V}$-shaped antenna is always lower, i.e., better than the UCA. In Fig 8(a), it is shown that the performance concerning the azimuth estimation is strictly linked to the number of sensors located on the orthogonal branch, i.e., on the coefficient $\alpha$. We observed that when the ratio $\alpha$ varies, the estimation performance concerning azimuth and elevation varies differently. When the one improves, the other deteriorates. For the value of $\alpha$ close to 1 , i.e., almost of the sensors located on the planar antenna, the estimation accuracy in terms of both the azimuth and elevation of the $\mathrm{V}$-shaped family is better than the one of the UCA.

- Unconditional observation model

Figs. 8(b) and 9(b) show that the performance concerning estimation of both azimuth and elevation are strongly dependent on the number of sensors located on the orthogonal branch, i.e., the coefficient $\alpha$. The link between $\alpha$ and the CRB under $H_{2}$ is more complicated than under $H_{1}$. When $\alpha$ decreases, then the CRB concerning azimuth estimation deteriorates, while the CRB concerning elevation estimation varies differently according to the DOA: it improves in some zone of 


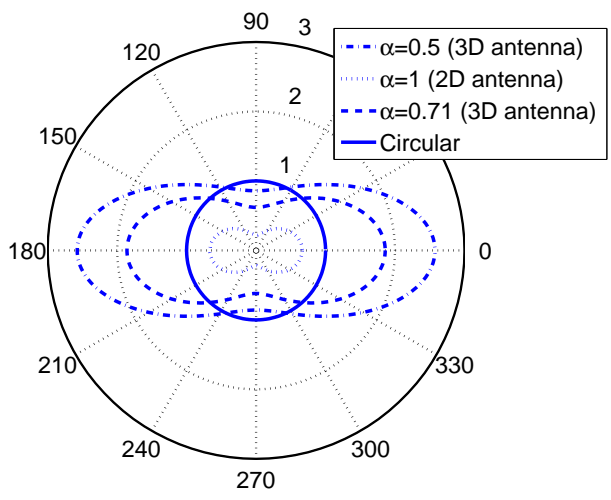

(a) Conditional observation model

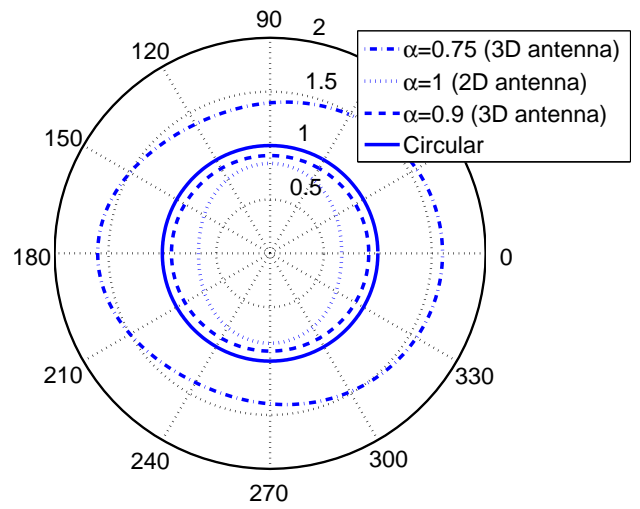

(b) Unconditional observation model

Fig. 8 Polar representation of the normalized CRB of azimuth for all values of azimuth angle, with different values of $\alpha, \Delta=60^{\circ}$, and $\theta=45^{\circ}$. The array has a single orthogonal branch.

DOA while worsens in the other zones. For the value of $\alpha$ close to 1 , the V-shaped family performs better in terms of both azimuth and elevation estimation than the UCA.

\subsection{Comparison of the estimation performance of the isotropic antennas}

We are interested in considering the case where our array attains the isotropic and uncoupling properties. We here compare the V-shaped isotropic array $\left(\Delta_{i s o}=\frac{\pi}{2}\right.$ under $H_{1}$ and $\Delta_{i s o}=\arccos \left(\frac{3\left(\alpha^{2}-1 / M^{2}\right)}{8 \alpha-3 \alpha^{2}+3 / M^{2}}\right)$ under $\left.H_{2}\right)$ with the classical isotropic UCA. As mentioned in the previous section, under $\mathrm{H}_{2}$, the $3 \mathrm{D} \mathrm{V}$-shaped extension 


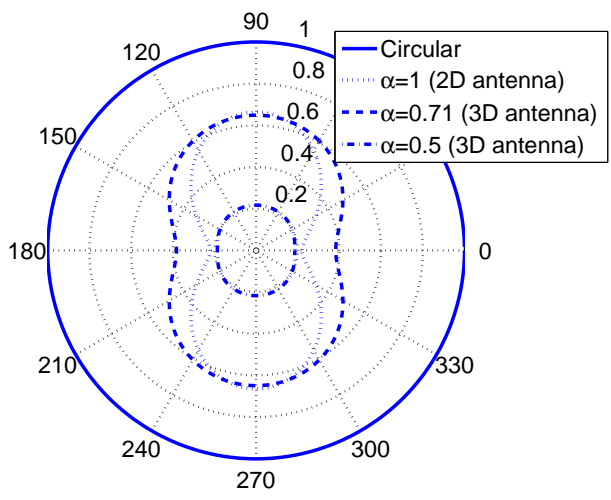

(a) Conditional observation model

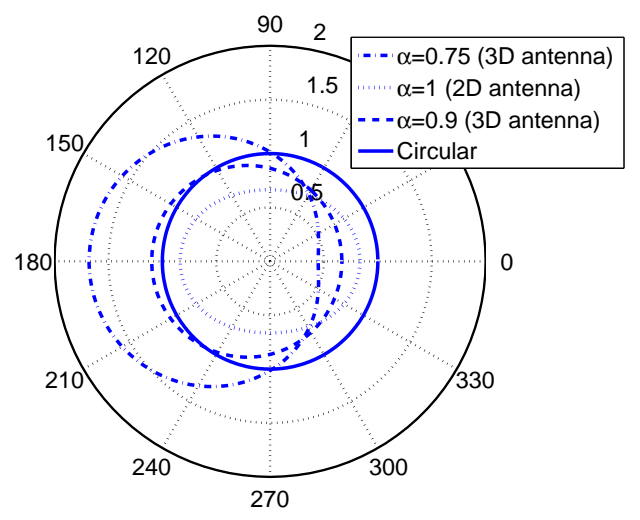

(b) Unconditional observation model

Fig. 9 Polar representation of the normalized CRB of elevation for all values of azimuth angle, with different values of $\alpha, \Delta=60^{\circ}$, and $\theta=45^{\circ}$. The array has a single orthogonal branch.

array becomes an isotropic array if there are two symmetric orthogonal branches. Therefore, under $H_{1}$, a $3 \mathrm{D} \mathrm{V}$-shaped array with a single orthogonal branch is used while under $\mathrm{H}_{2}$, a 3D V-shaped array with two symmetric orthogonal branches is used. We consider the ratio $K_{C}(M)$ (under $H_{1}$ ) or $K_{U}(M)$ (under $H_{2}$ ) between the CRB concerning the estimation of the azimuth of a family of V-shaped isotropic arrays and the UCA array. Thus, we have $K_{C}(M)=K_{U}(M)=\frac{C_{\phi \phi}^{2 D}}{C_{\phi \phi}^{U C A}}$ if $\alpha=1$ and $K_{C}(M)=K_{U}(M)=\frac{C_{\phi \phi}^{3 D}}{C_{\phi \phi}^{U C A}}$ if $\alpha<1$. Therefore, this fraction shows the gain in estimation of azimuth accuracy of the family of $\mathrm{V}$-shaped arrays w.r.t. to the UCA array. 


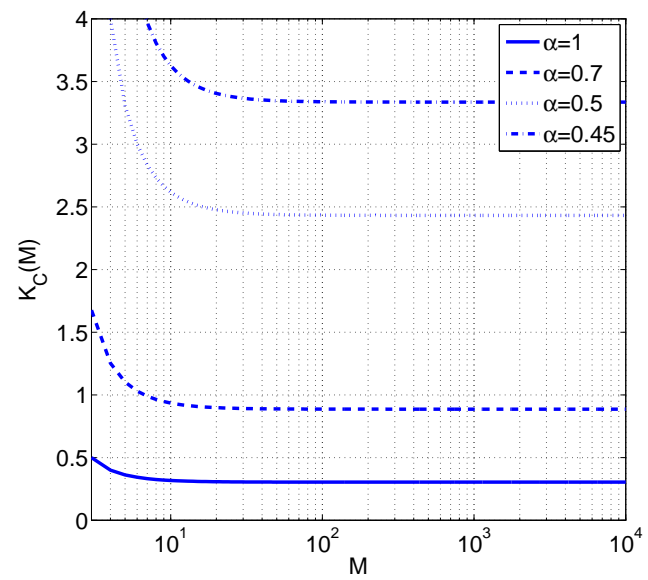

(a) Conditional observation model

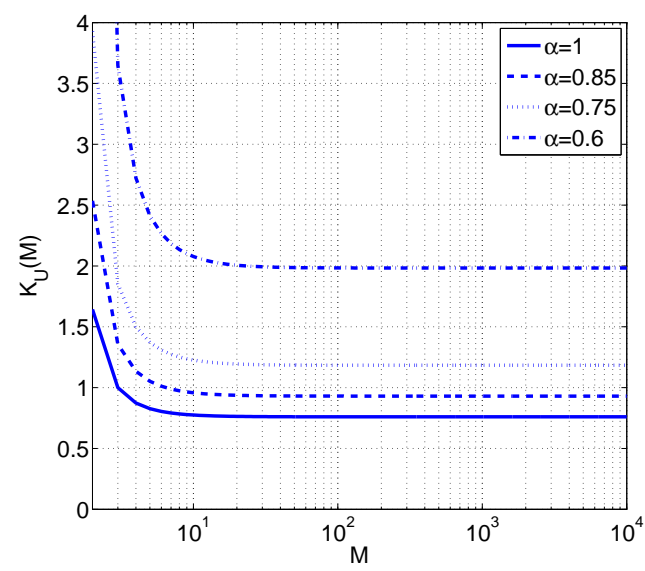

(b) Unconditional observation model

Fig. 10 Fraction $K(M)$ in term of the number of sensors $M$

\section{- Conditional observation model}

From (34), the ratio of CRB concerning azimuth of these antenna arrays is given by:

$$
\begin{aligned}
& K_{C}(M)=\frac{3}{\alpha\left(\alpha^{2} M^{2}-1\right) \sin ^{2} \frac{\pi}{M}} . \\
& \text { If } \alpha M>>1 \rightarrow K_{C}(M)=\frac{3}{\pi^{2} \alpha^{3}} .
\end{aligned}
$$

We can say that the V-shaped antenna array is better than the UCA array in terms of the estimation of azimuth if and only if the fraction $K_{C}(M)$ is smaller than 1. Fig. 10(a) shows that the 3D V-shaped isotropic antenna array is better than UCA array provided that the value of $\alpha$ satisfies: $0.76<\alpha<1$ and $M>6$. 
- Unconditional observation model

From (33), after some calculations, the ratio $K_{U}(M)$ is given by:

$$
\begin{aligned}
& K_{U}(M)=\frac{3\left(8 \alpha M^{2}-3 \alpha^{2} M^{2}+3\right)}{\sin ^{2} \frac{\pi}{M} \alpha\left(\alpha^{2} M^{2}-1\right)\left(8 \alpha M^{2}-6 \alpha^{2} M^{2}+6\right)} . \\
& \text { If } \alpha M>>1 \rightarrow K_{U}(M)=\frac{3(8-3 \alpha)}{\alpha^{3}(8-6 \alpha) \pi^{2}} .
\end{aligned}
$$

Fig. 10(b) shows that the $3 \mathrm{D} \mathrm{V}$-shaped isotropic antenna array is better than the UCA array if: $0.84<\alpha<1$ and $M>7$.

Table 1 'The azimuth estimation performance gain of 3D V-shaped isotropic antenna according to UCA'

\begin{tabular}{|c|c|c|c|c|c|}
\hline$\alpha$ & 1 & 0.9 & 0.8 & 0.7 & 0.6 \\
\hline$H_{1}$ assumption & 0.6959 & 0.5829 & 0.4060 & 0.1133 & -0.4081 \\
\hline$H_{2}$ assumption & 0.2399 & 0.1498 & -0.0393 & -0.3765 & -0.9838 \\
\hline
\end{tabular}

Tab. 1 shows the value of $1-K_{U}(M)$ and $1-K_{C}(M)$ w.r.t. $\alpha$. These values represent the gain concerning the azimuth estimation of the $3 \mathrm{D} \mathrm{V}$-shaped isotropic antenna array to the UCA array for a large number of sensors. We here want to find the value of $\alpha$, with which $1-K_{C}(M)>0$ under $H_{1}$ or $1-K_{U}(M)>0$ under $\mathrm{H}_{2}$ i.e., the $3 \mathrm{D} \mathrm{V}$-shaped antenna array has the better azimuth estimation accuracy than the UCA array. Under both $H_{1}$ and $H_{2}$ assumption, it is clear that, for all $\alpha>0.85$, the 3D $\mathrm{V}$-shaped isotropic array is always better than the UCA. Moreover, if $\alpha=1$ then the azimuth estimation accuracy of the V-shaped isotropic planar array is at least $20 \%$ better than the UCA array.

6.3 Comparison of the estimation performance between 2D and 3D antenna arrays

In the following, we compare the performance of estimation between the $3 \mathrm{D}$ and $2 \mathrm{D}$ arrays. The $\mathrm{V}$-shaped 2D antenna array has $M=7$ sensors (one at the origin with three other sensors on each branch). The $\mathrm{V}$-shaped 3D extension antenna array consisting of a single orthogonal branch is made also from $M=7$ sensors (one at the origin and two sensors on every three branches). It should be noted that taking some sensors from the planar array of the $2 \mathrm{D}$ antenna array to make the $3 \mathrm{D}$ antenna array will decrease the aperture and hence, reduce its performance. Therefore, using non ULA such as minimum redundancy, D-optimal, etc. instead of using ULA can maintain the aperture and also, the performance.

Fig. 11 shows the behaviors of $C_{\theta \theta}^{3 D}, C_{\phi \phi}^{3 D}, C_{\theta \theta}^{2 D}, C_{\phi \phi}^{2 D}$ in terms of the opening angle $\Delta$ varying from $0^{\circ}$ to $90^{\circ}$ under $H_{1}$ and $H_{2}$, and at $\phi=20^{\circ}$ and $\theta=70^{\circ}$. This is the scenario where the source is close to the plane of the array. Under both two assumptions, for the estimation of elevation, $\theta$, we can see that the $3 \mathrm{D}$ antenna array has always the better performance compared to the $2 \mathrm{D}$ antenna. However, concerning the azimuth estimation, the $3 \mathrm{D}$ array only has better performance than the $2 \mathrm{D}$ array if $\Delta<20^{\circ}$ under $H_{1}$ or $\Delta<12^{\circ}$ under $H_{2}$.

Fig. 12 shows the same curves, but values of $\phi$ and $\theta$ are respectively equal to $50^{\circ}$ and $30^{\circ}$. This is the scenario where the source is far from the plane of the 

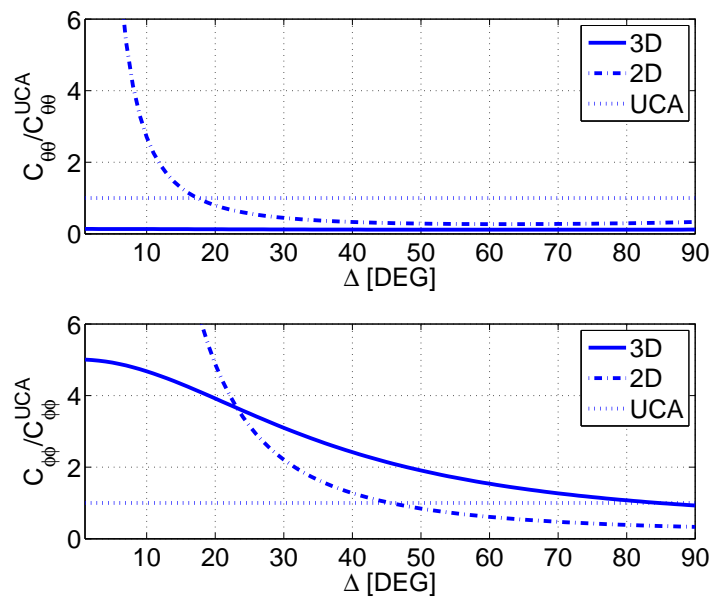

(a) Conditional observation model
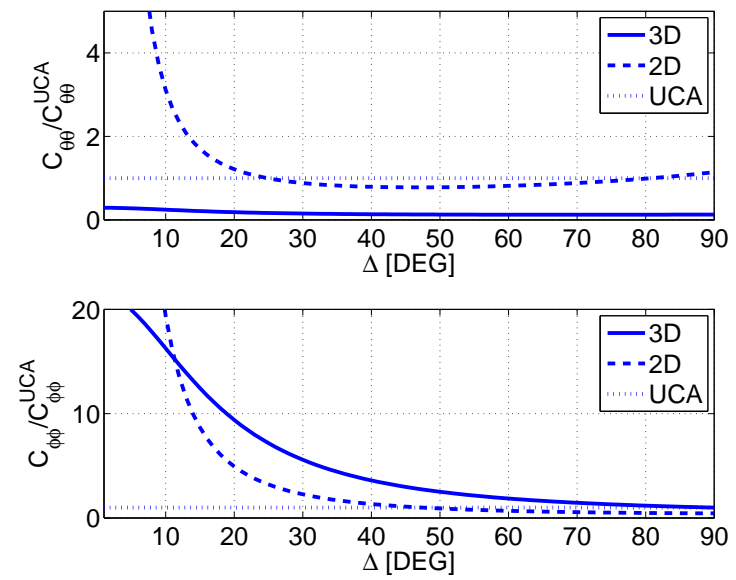

(b) Unconditional observation model

Fig. 11 The behavior of $C_{\theta \theta}^{3 D}, C_{\theta \theta}^{2 D}, C_{\phi \phi}^{3 D}$ and $C_{\phi \phi}^{2 D}$ normalized by the CRB of the UCA according to $\Delta$ at $\phi=20^{\circ}$ and $\theta=70^{\circ}$

antenna array. In this case, for both $H_{1}$ and $H_{2}$ assumptions, it should be better, contrary to intuition, to choose the $2 \mathrm{D}$ antenna array over a limited opening angle obtained numerically by solving $\max \left(C_{\phi \phi}^{3 D}=C_{\phi \phi}^{2 D}, C_{\theta \theta}^{3 D}=C_{\theta \theta}^{2 D}\right)$ as a function of $\Delta$.

Finally, by an exhaustive research over all three parameters: elevation, azimuth and opening angle, we found that concerning the elevation estimation, the 3D antenna array is always the better than the $2 \mathrm{D}$ antenna if the elevation is larger than a certain threshold $\theta_{0}$. 

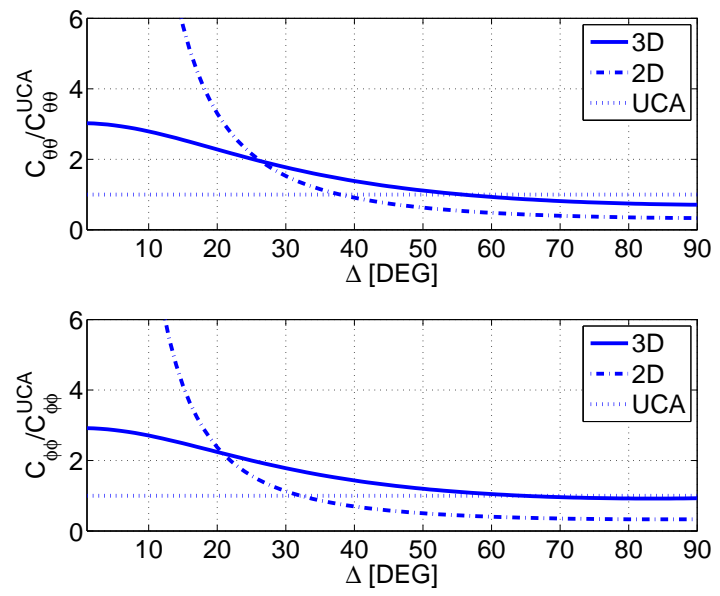

(a) Conditional observation model
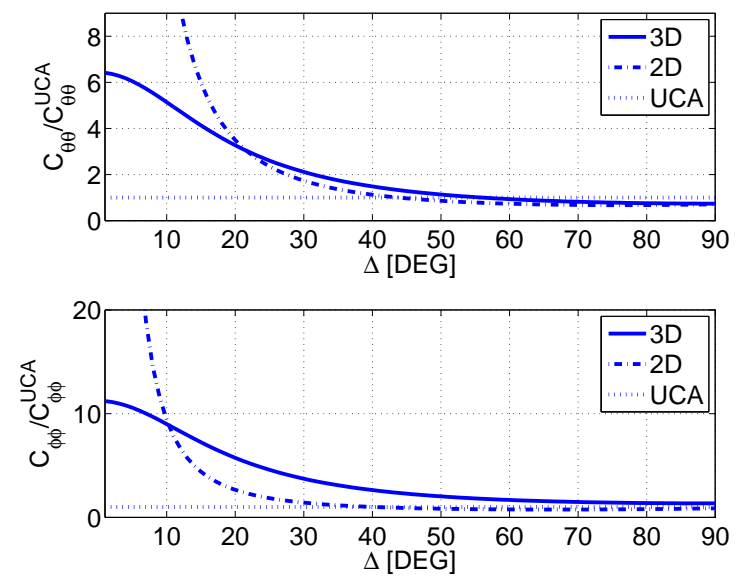

(b) Unconditional observation model

Fig. 12 The behavior of $C_{\theta \theta}^{3 D}, C_{\theta \theta}^{2 D}, C_{\phi \phi}^{3 D}$ and $C_{\phi \phi}^{2 D}$ normalized by the CRB of the UCA according to $\Delta$ at $\phi=50^{\circ}$ and $\theta=30^{\circ}$

\section{- Conditional observation model}

We can prove in this case that the threshold $\theta_{0}$ is about $62.2^{\circ}$ by solving

$$
\frac{C_{\theta \theta}^{3 D}}{C_{\theta \theta}^{2 D}}<1 \Leftrightarrow \theta>\arctan \sqrt{\max _{\Delta, \phi}\{\Gamma\}},
$$

where $\Gamma=\frac{\sin ^{2} \Delta\left(\left(M^{2}-1\right)-\alpha\left(\alpha^{2} M^{2}-1\right)\right)}{(1-\cos \Delta \cos 2 \phi) 4(1-\alpha)((1-\alpha) M+1)(2(1-\alpha) M+1)}, \alpha=\frac{N_{1}}{M}=\frac{5}{7}, M=7, \theta \in$ $\left[0^{\circ}, 90^{\circ}\right], \Delta \in\left(0^{\circ}, 180^{\circ}\right), \phi \in\left[0^{\circ}, 360^{\circ}\right]$. 
- Unconditional observation model

By numerical calculus, in the case where $\alpha=5 / 7$ and $M=7$, we obtain the threshold $\theta_{0} \simeq 65^{\circ}$.

\section{Conclusion}

In this paper, we derived the closed form expressions of the CRB for the estimation of azimuth and elevation of a far field, single source in both conditional and unconditional observation models where a planar array or its 3D extension is used. The $3 \mathrm{D}$ array extension here is made by adding one or two orthogonal branches to an arbitrary planar array. These CRB closed form expressions are used here as a useful tool in order to find the isotropy, uncoupling conditions, and the contribution of the third dimension to the estimation accuracy and also to introduce a comparison between conditional and unconditional observation models. Consequently, we showed that the $3 \mathrm{D}$ array overcomes the ambiguity problem of the planar (2D) array. Moreover, we found that there exists a family of array geometries with which the CRB can be expressed in the same term under both conditional and unconditional assumptions. Furthermore, at high signal to noise ratio or with a large number of sensors, the CRB expressions under the two assumptions become identical. In the following step, the CRB closed form expressions are then applied into several particular well-known array geometries such as: the V-shaped/ L-shaped array 3D extension, the uniform angular array. It is shown that the isotropy and uncoupling conditions of the 3D array under conditional and unconditional assumptions are different from each other. In particular, for the V-shaped arrays family, under the unconditional observation model, the opening angle $\Delta_{i s o}$ depends on the number of sensors located on the orthogonal branches while $\Delta_{i s o}=90^{\circ}$ is the desired value under the conditional assumption. Finally, through several simulations, we conclude that the performance of estimation of the 3D array strongly depends on the rate between the number of sensors located on the orthogonal branches and the total number of sensors $(\alpha)$. When this rate varies, the estimation concerning azimuth and elevation varies differently. In the other hand, by choosing a suitable rate ( $\alpha$ close to 1 ), the 3D array has the better performance than the classical UCA concerning both azimuth and elevation estimation for the same number of sensors. It should be noted that, for a constant number of sensors, adding the 3D branch will decrease the aperture of the antenna, therefore, deteriorate the estimation performance.

\section{Appendix}

A Proof of Eqn. (15)

Let us note that the sensors located on the $x O y$ plane are such that $\xi_{i}=\frac{\pi}{2}$, while the sensors located on the orthogonal axe are such that $\xi_{i}=0$. After some calculation, from (7), it easy 
to obtain the elements of the Fisher Information Matrix:

$$
\begin{aligned}
\frac{[\mathbf{F I M}(\boldsymbol{\Theta})]_{11}}{C_{S N R}} & =\sum_{i=1}^{M} \rho_{i}^{2}\left(\cos \theta \sin \xi_{i} \cos \left(\phi-\varphi_{i}\right)-\cos \xi_{i} \sin \theta\right)^{2} \\
& =\sum_{i=1}^{N_{1}} \rho_{1, i}^{2} \cos ^{2} \theta \cos ^{2}\left(\phi-\varphi_{i}\right)+\sum_{i=N_{1}+1}^{M} \rho_{2, i}^{2} \sin ^{2} \theta \\
& =\frac{\cos ^{2} \theta}{4}\left(e^{2 j \phi} S_{12}^{*}+e^{-2 j \phi} S_{12}+2 S_{10}\right)+\sin ^{2} \theta S_{20} \\
& =\frac{1}{2} \cos ^{2} \theta\left(\Re\left\{e^{-2 j \phi} S_{12}\right\}+S_{10}\right)+\sin ^{2} \theta S_{20}, \\
\frac{[\mathbf{F I M}(\boldsymbol{\Theta})]_{22}}{C_{S N R}} & =\sum_{i=1}^{M} \rho_{i}^{2}\left(\sin \theta \sin \xi_{i} \sin \left(\phi-\varphi_{i}\right)\right)^{2} \\
& =\sin ^{2} \theta \sum_{i=1}^{N_{1}} \rho_{1, i}^{2} \sin ^{2}\left(\phi-\varphi_{i}\right) \\
& =-\frac{\sin ^{2} \theta}{4}\left(e^{2 j \phi} S_{12}^{*}+e^{-2 j \phi} S_{12}-2 S_{10}\right) \\
& \sin ^{2} \theta\left(\Re\left\{e^{-2 j \phi} S_{12}\right\}-S_{10}\right),
\end{aligned}
$$

and

$$
\begin{aligned}
\frac{[\mathbf{F I M}(\boldsymbol{\Theta})]_{12}}{C_{S N R}} & =-\sum_{i=1}^{M} \rho_{i}^{2} \sin \theta \sin \xi_{i} \sin \left(\phi-\varphi_{i}\right)\left(\cos \theta \sin \xi_{i} \cos \left(\phi-\varphi_{i}\right)-\cos \xi_{i} \sin \theta\right) \\
& =-\sin \theta \cos \theta \sum_{i=1}^{N_{1}} \rho_{1, i}^{2} \sin \left(\phi-\varphi_{i}\right) \cos \left(\phi-\varphi_{i}\right) \\
& =-\frac{1}{8 j} \sin 2 \theta\left(e^{2 j \phi} S_{12}^{*}-e^{-2 j \phi} S_{12}\right) \\
& =\frac{1}{4} \sin 2 \theta \Im\left\{e^{-2 j \phi} S_{12}\right\} .
\end{aligned}
$$

The FIM determinant is given by:

$$
\begin{aligned}
& \frac{\operatorname{det}[\mathbf{F I M}(\boldsymbol{\Theta})]}{C_{S N R}^{2}}=\frac{[\mathbf{F I M}(\boldsymbol{\Theta})]_{11}[\mathbf{F I M}(\boldsymbol{\Theta})]_{22}-[\mathbf{F I M}(\boldsymbol{\Theta})]_{12}[\mathbf{F I M}(\boldsymbol{\Theta})]_{21}}{C_{S N R}^{2}} \\
= & \left(\frac{\cos ^{2} \theta}{4}\left(e^{2 j \phi} S_{12}^{*}+e^{-2 j \phi} S_{12}+2 S_{10}\right)+\sin ^{2} \theta S_{20}\right)\left(-\frac{\sin ^{2} \theta}{4}\left(e^{2 j \phi} S_{12}^{*}+e^{-2 j \phi} S_{12}-2 S_{10}\right)\right) \\
& -\left(-\frac{1}{8 j} \sin 2 \theta\left(e^{2 j \phi} S_{12}^{*}-e^{-2 j \phi} S_{12}\right)\right)^{2} \\
= & \frac{\sin ^{2} 2 \theta}{64}\left(4 S_{10}^{2}-\left(e^{2 j \phi} S_{12}^{*}+e^{-2 j \phi} S_{12}\right)^{2}\right)-\frac{\sin ^{4} \theta}{4} S_{20}\left(e^{2 j \phi} S_{12}^{*}+e^{-2 j \phi} S_{12}-2 S_{10}\right) \\
& +\frac{\sin ^{2} 2 \theta}{64}\left(e^{2 j \phi} S_{12}^{*}-e^{-2 j \phi} S_{12}\right)^{2} \\
= & \frac{\sin ^{2} 2 \theta}{64}\left(4 S_{10}^{2}-4\left|S_{12}\right|^{2}\right)+\frac{\sin ^{4} \theta}{4} S_{20}\left(2 S_{10}-2 \Re\left\{e^{-2 j \phi} S_{12}\right\}\right) \\
= & \frac{\sin ^{2} \theta}{4}\left(\cos ^{2} \theta\left(S_{10}^{2}-\left|S_{12}\right|^{2}\right)+2 \sin ^{2} \theta S_{20}\left(S_{10}-\Re\left\{e^{-2 j \phi} S_{12}\right\}\right)\right) .
\end{aligned}
$$


B Proof of Eqn. (16) and Eqn. (17)

In the same way as for conditional case, from (13), we have

$$
\begin{aligned}
& \frac{[\mathbf{F I M}(\Theta)]_{1,1}}{U_{S N R}}= \\
= & \sum_{i=1}^{M} \rho_{i}^{2}\left(\cos \theta \sin \xi_{i} \cos \left(\phi-\varphi_{i}\right)-\cos \xi_{i} \sin \theta\right)^{2} \\
& -\frac{1}{M}\left(\sum_{i=1}^{M} \rho_{i}\left(\cos \theta \sin \xi_{i} \cos \left(\phi-\varphi_{i}\right)-\cos \xi_{i} \sin \theta\right)\right)^{2} \\
= & \cos ^{2} \theta \sum_{i=1}^{N_{1}} \rho_{1, i}^{2} \cos ^{2}\left(\phi-\varphi_{i}\right)+\sin ^{2} \theta \sum_{i=N_{1}+1}^{M} \rho_{2, i}^{2} \\
& -\frac{1}{M}\left(\cos \theta \sum_{i=1}^{N_{1}} \rho_{1, i} \cos \left(\phi-\varphi_{i}\right)-\sin \theta \sum_{i=N_{1}+1}^{M} \rho_{2, i}\right)^{2} \\
= & \frac{1}{4} \cos ^{2} \theta\left(S_{12} e^{-2 j \phi}+S_{12}^{*} e^{2 j \phi}+2 S_{10}\right)+\sin ^{2} \theta S_{20} \\
& -\frac{1}{M}\left(\frac{\cos ^{2} \theta}{4}\left(S_{11}^{2} e^{-2 j \phi}+S_{11}^{2 *} e^{2 j \phi}+2\left|S_{11}\right|^{2}\right)\right. \\
& \left.\quad+\sin ^{2} \theta S_{23}^{2}-\frac{\sin 2 \theta}{2}\left(S_{11} e^{-j \phi}+S_{11}^{*} e^{j \phi}\right) S_{23}\right) \\
= & \frac{1}{2} \cos ^{2} \theta\left(S_{10}-\frac{\left|S_{11}\right|^{2}}{M}+\Re\left\{e^{-2 j \phi}\left(S_{12}-\frac{S_{11}^{2}}{M}\right)\right\}\right) \\
& +\sin ^{2} \theta\left(S_{20}-\frac{S_{23}^{2}}{M}\right)+\frac{\sin 2 \theta}{M} S_{23} \Re\left\{e^{-j \phi} S_{11}\right\},
\end{aligned}
$$

$$
\begin{aligned}
& \frac{[\mathbf{F I M}(\boldsymbol{\Theta})]_{2,2}}{U_{S N R}}=\sum_{i=1}^{M} \rho_{i}^{2} \sin ^{2} \theta \sin ^{2} \xi_{i} \sin ^{2}\left(\phi-\varphi_{i}\right)-\frac{1}{M}\left(\sum_{i=1}^{M} \rho_{i} \sin \theta \sin \xi_{i} \sin \left(\phi-\varphi_{i}\right)\right)^{2} \\
& =\sin ^{2} \theta \sum_{i=1}^{N_{1}} \rho_{p, i}^{2} \sin ^{2}\left(\phi-\varphi_{i}\right)-\frac{\sin ^{2} \theta}{M}\left(\sum_{i=1}^{N_{1}} \rho_{1, i} \sin \left(\phi-\varphi_{i}\right)\right)^{2} \\
& =-\frac{1}{4} \sin ^{2} \theta\left(e^{-2 j \phi} S_{12}+e^{2 j \phi} S_{12}^{*}-2 S_{10}\right)+\frac{\sin ^{2} \theta}{4 M}\left(e^{2 j \phi} S_{11}^{2 *}+e^{-2 j \phi} S_{11}^{2}-2\left|S_{11}\right|^{2}\right) \\
& =\frac{1}{2} \sin ^{2} \theta\left(S_{10}-\frac{\left|S_{11}\right|^{2}}{M}-\Re\left\{e^{-2 j \phi}\left(S_{12}-\frac{S_{11}^{2}}{M}\right)\right\}\right),
\end{aligned}
$$

and

$$
\begin{aligned}
& \frac{[\mathbf{F I M}(\boldsymbol{\Theta})]_{1,2}}{U_{S N R}}=-\sum_{i=1}^{M} \rho_{i} \sin \theta \sin \xi_{i} \sin ^{2}\left(\phi-\varphi_{i}\right)\left(\cos \theta \sin \xi_{i} \cos \left(\phi-\varphi_{i}\right)-\cos \xi_{i} \sin \theta\right) \\
& \quad+\frac{1}{M} \sum_{i=1}^{M} \rho_{i}\left(\cos \theta \sin \xi_{i} \cos \left(\phi-\varphi_{i}\right)-\cos \xi_{i} \sin \theta\right) \sum_{i=1}^{M} \rho_{i} \sin \theta \sin \xi_{i} \sin \left(\phi-\varphi_{i}\right) \\
= & -\sin \theta \cos \theta \sum_{i=1}^{N_{1}} \rho_{1, i}^{2} \sin \left(\phi-\varphi_{i}\right) \cos \left(\phi-\varphi_{i}\right) \\
& +\frac{1}{M} \sin \theta\left(\cos \theta \sum_{i=1}^{N_{1}} \rho_{1, i} \cos \left(\phi-\varphi_{i}\right)-\sin \theta \sum_{i=N_{1}+1}^{M} \rho_{2, i}\right) \sum_{i=1}^{N_{1}} \rho_{1, i} \sin \left(\phi-\varphi_{i}\right) \\
= & -\frac{\sin \theta \cos \theta}{4 j}\left(e^{2 j \phi} S_{12}^{*}-e^{-2 j \phi} S_{12}\right) \\
& +\frac{\sin \theta}{2 j M}\left(\frac{1}{2} \cos \theta\left(e^{j \phi} S_{11}+e^{-j \phi} S_{11}^{*}\right)-\sin \theta S_{23}\right)\left(e^{j \phi} S_{11}^{*}-e^{-j \phi} S_{11}\right) \\
= & \frac{\sin \theta \cos \theta}{2} \Im\left\{e^{-2 j \phi}\left(S_{12}-\frac{S_{11}^{2}}{M}\right)\right\}+\frac{\sin ^{2} \theta}{M} S_{23} \Im\left\{e^{-j \phi} S_{11}\right\} .
\end{aligned}
$$


The FIM determinant is given by:

$$
\begin{aligned}
& \frac{\operatorname{det}[\mathbf{F I M}(\boldsymbol{\Theta})]}{U_{S N R}^{2}}=\frac{[\mathbf{F I M}(\boldsymbol{\Theta})]_{1,1}[\mathbf{F I M}(\boldsymbol{\Theta})]_{2,2}-[\mathbf{F I M}(\boldsymbol{\Theta})]_{1,2}[\mathbf{F I M}(\boldsymbol{\Theta})]_{2,1}}{U_{S}^{2}} \\
& =\left(\frac{1}{2} \cos ^{2} \theta\left(S_{10}-\frac{\left|S_{11}\right|^{2}}{M}+\Re\left\{e^{-2 j \phi}\left(S_{12}-\frac{S_{11}^{2}}{M}\right)\right\}\right)\right. \\
& \left.+\sin ^{2} \theta\left(S_{20}-\frac{S_{23}^{2}}{M}\right)+\frac{\sin 2 \theta}{M} S_{23} \Re\left\{e^{-j \phi} S_{11}\right\}\right) \\
& \times\left(\frac{1}{2} \sin ^{2} \theta\left(S_{10}-\frac{\left|S_{11}\right|^{2}}{M}-\Re\left\{e^{-2 j \phi}\left(S_{12}-\frac{S_{11}^{2}}{M}\right)\right\}\right)\right) \\
& -\left(\frac{1}{2} \sin \theta \cos \theta \Im\left\{e^{-2 j \phi}\left(S_{12}-\frac{S_{11}^{2}}{M}\right)\right\}+\frac{1}{M} \sin ^{2} \theta S_{23} \Im\left\{e^{-j \phi} S_{11}\right\}\right)^{2} \\
& =\frac{1}{4} \sin ^{2} \theta \cos ^{2} \theta\left(\left(S_{10}-\frac{\left|S_{11}\right|^{2}}{M}\right)^{2}-\Re^{2}\left\{e^{-2 j \phi}\left(S_{12}-\frac{S_{11}^{2}}{M}\right)\right\}\right) \\
& +\frac{1}{2} \sin ^{2} \theta\left(S_{10}-\frac{\left|S_{11}\right|^{2}}{M}-\Re\left\{e^{-2 j \phi}\left(S_{12}-\frac{S_{11}^{2}}{M}\right)\right\}\right) \\
& \times\left(\sin ^{2} \theta\left(S_{20}-\frac{S_{23}^{2}}{M}\right)+\frac{\sin 2 \theta}{M} S_{23} \Re\left\{e^{-j \phi} S_{11}\right\}\right) \\
& -\frac{1}{4} \sin ^{2} \theta \cos ^{2} \theta \Im^{2}\left\{e^{-2 j \phi}\left(S_{12}-\frac{S_{11}^{2}}{M}\right)\right\}-\frac{1}{M^{2}} \sin ^{4} \theta S_{23}^{2} \Im^{2}\left\{e^{-j \phi} S_{11}\right\} \\
& -\frac{1}{M} \sin ^{3} \theta \cos \theta \Im\left\{e^{-2 j \phi}\left(S_{12}-\frac{S_{11}^{2}}{M}\right)\right\} S_{23} \Im\left\{e^{-j \phi} S_{11}\right\} \\
& =\frac{1}{4} \sin ^{2} \theta \cos ^{2} \theta\left(\left(S_{10}-\frac{\left|S_{11}\right|^{2}}{M}\right)^{2}-\left|S_{12}-\frac{S_{11}^{2}}{M}\right|^{2}\right) \\
& +\sin ^{4} \theta\left(\frac{1}{2}\left(S_{20}-\frac{S_{23}^{2}}{M}\right)\left(S_{10}-\frac{\left|S_{11}\right|^{2}}{M}-\Re\left\{e^{-2 j \phi}\left(S_{12}-\frac{S_{11}^{2}}{M}\right)\right\}\right)-\frac{1}{M^{2}} S_{23}^{2} \Im^{2}\left\{e^{-j \phi} S_{11}\right\}\right) \\
& +\frac{S_{23}}{M} \sin ^{3} \theta \cos \theta \\
& \times\left(\Re\left\{e^{-j \phi} S_{11}\right\}\left(S_{10}-\frac{\left|S_{11}\right|^{2}}{M}-\Re\left\{e^{-2 j \phi}\left(S_{12}-\frac{S_{11}^{2}}{M}\right)\right\}\right)\right. \\
& \left.-\Im\left\{e^{-j \phi} S_{11}\right\} \Im\left\{e^{-2 j \phi}\left(S_{12}-\frac{S_{11}^{2}}{M}\right)\right\}\right) \\
& =\frac{1}{4} \sin ^{2} \theta \cos ^{2} \theta\left(\left(S_{10}-\frac{\left|S_{11}\right|^{2}}{M}\right)^{2}-\left|S_{12}-\frac{S_{11}^{2}}{M}\right|^{2}\right) \\
& +\sin ^{4} \theta\left(\frac{1}{2} S_{20}\left(S_{10}-\frac{\left|S_{11}\right|^{2}}{M}-\Re\left\{e^{-2 j \phi}\left(S_{12}-\frac{S_{11}^{2}}{M}\right)\right\}\right)-\frac{S_{23}^{2}}{2 M}\left(S_{10}-\Re\left\{e^{-2 j \phi} S_{12}\right\}\right)\right) \\
& +\frac{S_{23} \sin ^{2} \theta \sin 2 \theta}{2 M}\left(S_{10} \Re\left\{e^{-j \phi} S_{11}\right\}-\Re\left\{e^{-j \phi} S_{12} S_{11}^{*}\right\}\right) \text {. }
\end{aligned}
$$

\section{Proof of Eqn. (18)}

Note that the sensors located on the $x O y$ plane are such that $\xi_{i}=\frac{\pi}{2}$, while the sensors located on the first orthogonal axe have $\xi_{i}=0$, and the sensors located on the second orthogonal axe are such that $\xi_{i}=\pi$. In the same way as we prove Eqn. (16) and (17), with the assumption that the two orthogonal branches are symmetric, it leads to:

$$
\sum_{i=1}^{N_{2}} \rho_{2, i} \cos \xi_{i}=\sum_{i=1}^{\frac{N_{2}}{2}} \rho_{2, i} \cos 0+\sum_{i=\frac{N_{2}}{2}+1}^{N_{2}} \rho_{2, i} \cos \pi=\sum_{i=1}^{\frac{N_{2}}{2}} \rho_{2, i}-\sum_{i=\frac{N_{2}}{2}+1}^{N_{2}} \rho_{2, i}=0 .
$$

Finally it is easy obtain (18) from Eqn. (16) and (17) by letting $S_{23}=0$.

Acknowledgements This project was funded by both région Île-de-France and Digiteo Research Park 


\section{References}

1. A. Manikas, Differential Geometry in Array Processing, Imperial College Press, 2004.

2. L. C. Godara, A. Cantoni, Uniqueness and linear independence of sterring vectors in array space, J. Acoust. Soc. Amer. 70 (2) (1981) 467- 475.

3. K. C. Tan, S. S. Goh, E. C. Tan, A study of the rank-ambiguilty issues in direction-ofarrival estimation, IEEE Trans. Signal Processing 44 (4) (1996) 880-887.

4. J. T. H. Lo, S. L. M. Jr, Observability conditions for multiple signal direction finding and array sensor localization, IEEE Trans. Signal Processing 40 (11) (1992) 2641- 2650.

5. M. Gavish, A. J. Weiss, Array geometry for ambiguity resolution in direction finding, IEEE Trans. Antennas Propagat. 44 (6) (1991) 143- 146.

6. H. L. VanTrees, Detection, Estimation and Modulation Theory: Optimum Array Processing, Vol. 4, Wiley, New York, 2002.

7. P. Stoica, R. Moses, Spectral Analysis of Signals, Prentice Hall, NJ, 2005.

8. U. Baysal, R. L. Moses, On the geometry of isotropic arrays, IEEE Trans. Signal Processing 51 (6) (2003) 1469-1477.

9. D. Sengupta, T. Smith, R. Larson, Radiation characteristics of a spherical array of circularly polarized elements, IEEE Trans. Antennas Propagat. 16 (1) (1968) 2- 7.

10. P. Stoica, A. Nehorai, MUSIC, maximum likelihood and the Cramér-Rao bound, IEEE Trans. Acoust., Speech, Signal Processing 37 (1989) 720-741.

11. P. Stoica, A. Nehorai, Performances study of conditional and unconditional direction of arrival estimation, IEEE Trans. Acoust., Speech, Signal Processing 38 (1990) 1783-1795.

12. A. Renaux, P. Foster, E. Chaumette, P. Larzabal, On the High-SNR Conditional Maximum-Likelihood estimator full statistical characterization, IEEE Trans. Signal Processing 54 (12) (2006) 4840- 4843.

13. A. Renaux, P. Foster, E. Boyer, P. Larzabal, Unconditional Maximum Likelihood performance at finite number of samples and high Signal-to-Noise Ratio, IEEE Trans. Signal Processing 55 (5) (2007) 2358-2364.

14. Y. Hua, T. K. Sarkar, A note on the Cramér-Rao bound for 2-D direction finding based on 2-D array, IEEE Trans. Signal Processing 39 (5) (1991) 1215- 1218.

15. R. O. Nielsen, Azimuth and elevation angle estimation with a three dimensional array, IEEE J. Oceanic Eng. 19 (1) (1994) 84-86.

16. A. Mirkin, L. H. Sibul, Cramér-Rao bounds on angle estimation with a two-dimensional array, IEEE Trans. Signal Processing 39 (1991) 515-517.

17. B. Yang, J. Scheuing, Cramér-Rao bound and optimum sensor array for source localization from the differences of arrival, in: Proc. IEEE Int. Conf. Acoust., Speech, Signal Processing, Vol. 4, Philadenphia, USA, 2005, pp. 961-964.

18. K. W. K. Lui, H. C. So, A study of two-dmensional sensor placement using time-differenceof-arrival measurements, Digital Signal Processing 19 (2009) 650- 659.

19. U. Oktel, R. L. Moses, A Bayesian approach to array geometry design, IEEE Trans. Signal Processing 53 (5) (2005) 1919- 1923.

20. H. Gazzah, S. Marcos, Cramér-Rao bounds for antenna array design, IEEE Trans. Signal Processing 54 (1) (2006) 336-345.

21. H. Gazzah, K. Abed-Meraim, Optimum ambiguity free directional and omni directional planar antenna arrays for DOA estimation, IEEE Trans. Signal Processing 57 (10) (2009) 3942-3953.

22. A. Ferréol, P. Chevalier, High resolution direction finding: from performance toward antenna array optimization -the mono-source case, in: Proc. European Signal Processing Conference, Glasgow, Scotland, 2009, pp. 1973-1977.

23. B. Ottersten, M. Viberg, P. Stoica, A. Nehorai, Exact and large sample maximum likelihood techniques for parameter estimation and detection in array processing, in: S. Haykin, J. Litva, T. J. Shepherd (Eds.), Radar Array Processing, Springer-Verlag, Berlin, 1993, Ch. 4, pp. 99-151.

24. J. Li, R. T. Compton, Maximum likelihood angle estimation for signals with known waveforms, IEEE Trans. Signal Processing 41 (1993) 2850-2862.

25. M. Cedervall, R. L. Moses, Efficient maximum likelihood DOA estimation for signals with known waveforms in presence of multipath, IEEE Trans. Signal Processing 45 (1997) 808811.

26. J. Li, B. Halder, P. Stoica, M. Viberg, Computationally efficient angle estimation for signals with known waveforms, IEEE Trans. Signal Processing 43 (1995) 2154-2163. 
27. A. Leshem, A.-J. van der Veen, Direction-of-arrival estimation for constant modulus signals, IEEE Trans. Signal Processing 47 (11) (1999) 3125 - 3129.

28. Y. H. Choi, Unified approach to Cramer-Rao bounds in direction estimation with known signal structures, Signal Processing 84 (10) (2004) 1875 - 1882.

29. Y. Hua, T. K. Sarkar, D. D. Weiner, An L-shaped array for estimating 2D directions of wave arrival, IEEE Trans. Antennas Propagat. 39 (1991) 143- 146.

30. T. Filik, T. E. Tuncer, Uniform and nonuniform V-shaped isotropic planar arrays, in: Proc. Sensor Array and Multichannel Signal Processing Workshop, Darmstadt, Germany, 2008, pp. 99-103.

31. S. M. Kay, Fundamentals of Statistical Signal Processing, Vol. 1, Prentice Hall, NJ, 1993.

32. A. V. D. Bos, A Cramer Rao lower bound for complex parameters, IEEE Trans. Acoust., Speech, Signal Processing 42 (1994) 2859.

33. B. Porat, B. Friedlander, Analysis of the asymptotic relative efficiency of the MUSIC algorithm, IEEE Trans. Acoust., Speech, Signal Processing 36 (4) (1988) 532-544.

34. A. T. Moffet, Minimum redundancy linear arrays, IEEE Trans. Antennas Propagat. 16 (1968) 172-175.

35. X. Huang, J. P. Reilly, M. Wong, Optimal design of linear array of sensors, in: Proc. IEEE Int. Conf. Acoust., Speech, Signal Processing, Vol. 2, Toronto, Ont., Canada, 1991, pp. 1405-1408. 